\title{
Covalent Functionalization of Nickel Phosphide Nanocrystals with Aryl-Diazonium Salts
}

\author{
Ian A. Murphy, ${ }^{a}$ Peter S. Rice, ${ }^{b}$ Madison Monahan, ${ }^{a}$ Leo P. Zasada, ${ }^{a}$ Elisa M. Miller, ${ }^{c}$ Simone \\ Raugei, ${ }^{b}$ Brandi M. Cossairt ${ }^{\mathrm{a} *}$

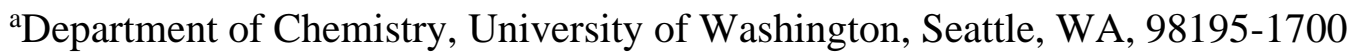 \\ ${ }^{b}$ Center for Molecular Electrocatalysis, Pacific Northwest National Laboratory, Richland, WA, \\ 99352 \\ ${ }^{c}$ National Renewable Energy Laboratory, 15013 Denver West Parkway, Golden, CO, 80401 \\ *cossairt@uw.edu
}

\begin{abstract}
Covalent functionalization of $\mathrm{Ni}_{2} \mathrm{P}$ nanocrystals was demonstrated using aryl-diazonium salts. Spontaneous adsorption of aryl functional groups was observed, with surface coverages ranging from $20-96 \%$ depending on the native reactivity of the salt as determined by the aryl substitution pattern. Increased coverage was possible for low reactivity species using a sacrificial reductant. Functionalization was confirmed using thermogravimetric analysis, FTIR and X-ray photoelectron spectroscopy. The structure and energetics of this nanocrystal electrocatalyst system, as a function of ligand coverage, was explored with density functional theory calculations. The Hammett parameter of the surface functional group was found to linearly correlate with the change in $\mathrm{Ni}$ and $\mathrm{P}$ core-electron binding energies and the nanocrystal's work-function. The electrocatalytic activity and stability of the functionalized nanocrystals for hydrogen evolution were also improved when compared to the unfunctionalized material, but a simple trend based on electrostatics was not evident.
\end{abstract}

\section{TOC Graphic}

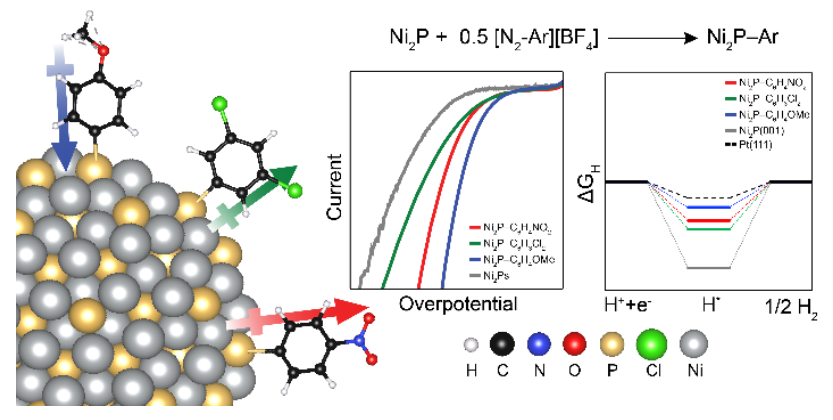

\section{Introduction}

Great progress has been made over the past few decades in the development and deployment of renewable energy technologies. This global effort has positioned sustainably generated electricity to, in some cases, be cheaper than carbon-based competitors. ${ }^{1}$ While renewably-sourced electricity will be a key factor in decarbonizing the power and light passenger transportation sectors, there is still significant work to be done in decarbonizing other carbon intensive sectors such as industrial transportation and chemical commodities. ${ }^{2-4}$ To achieve the same emissions reductions in these sectors, we must fundamentally alter the way we produce and recycle small molecule chemical feedstocks such as $\mathrm{H}_{2}, \mathrm{O}_{2}, \mathrm{~N}_{2}$, and $\mathrm{CO}_{2}$. The key component to enabling this 
kind of sustainable circular economy is the development of efficient, selective, and economically viable (photo)electrocatalysts. ${ }^{5}$

Today, the most active catalytic materials are scarce noble metals such as platinum, palladium, and iridium. Specifically in the case of the hydrogen evolution reaction (HER), the high activity of these materials has been ascribed to their ideal metal-hydride $(\mathrm{M}-\mathrm{H})$ bond dissociation free energies (BDFEs), their diffuse frontier orbitals that span the Fermi level, and their stability in highly acidic and basic media. ${ }^{6-8}$ The relationship between these types of "descriptors" and the intrinsic activity of a catalyst is still being investigated and qualified by many computational and electroanalytical groups. ${ }^{5,9-15}$ Descriptor-based models for understanding catalytic activity have led to the discovery of earth-abundant catalytic materials with relatively high intrinsic activity. ${ }^{5}$ Initial efforts in this space were focused, in some instances, on the investigation of molybdenum disulfide due to its solution processability, low toxicity, and long history of use as a hydrodesulfurization catalyst. ${ }^{16-19}$ This same line of theory driven inquiry led to the investigation of transition metal phosphides as a new class of materials with excellent catalytic activity for HER and high stability in acidic media. Research efforts over the past decade have explored many aspects of these materials including synthetic methodology and mechanisms ${ }^{20,21,30,22-29}$, the effects of varying atomic composition ${ }^{31-34}$, nano-structuring ${ }^{35-37}$, and computational modeling of their catalytically active surface sites. ${ }^{31,38-44}$

Despite great progress in developing this class of materials, earth abundant alternatives still present orders of magnitude lower activity than their precious metal counterparts. This begs the question many researchers are now investigating, "what are we missing in the current descriptorbased models?" If we can already achieve thermoneutral surface binding sites, maybe the answer lies just beyond the surface in the secondary and outer substrate coordination spheres?

More than a century of metal-based molecular (homogeneous) catalyst development highlighted the pivotal role of ligands in determining the chemical properties of a metal center, such as redox potential and metal-substrate BDFE. ${ }^{9,45-48}$ It is also clear that the way ligands interact with substrate and solvent, through secondary and outer-coordination sphere effects, have a dramatic influence on the rate of catalytic turnover and product selectivity. ${ }^{49-52} \mathrm{We}$ propose hybrid inorganic nanomaterials with tailor-made organic surface ligands could offer a strategy to translate the lessons learned in molecular systems to nanoparticles and represent a vast and underexplored parameter space in heterogeneous electrocatalysis. ${ }^{53-55}$

Previous studies from our laboratory have demonstrated the importance of the interface between heterogeneous nanoparticle surfaces and the bulk solvent. ${ }^{56,57}$ Through this work, for instance, we found that the predominant reason long chain aliphatic ligands hinder catalytic activity in aqueous media is because of the poor interface formed between the non-polar surface and the polar electrolyte. Just as aliphatic ligands make a poor interface with polar solvents, it has been shown that polar ligands that are able to hydrogen bond with a polar solvent can induce some degree of local order near the surface of a nanoparticle. ${ }^{58}$ We also know that surface ligands are able to shift the Fermi level of a nanomaterial based on their electron withdrawing/donating ability by inducing or enhancing the surface dipole. ${ }^{59-61}$ These isolated studies provide, in concert, a road map to tune a variety of catalytically relevant parameters in heterogenous nanoparticle catalysts.

In colloidal nanoparticle systems, ligand chemistry is most commonly explored using dative Lewis acid/base interactions between surface metal cation sites and electron rich binding groups such as amines, carboxylates, and phosphonates. ${ }^{62,63}$ Well understood mechanisms for ligand exchange have been developed based on concentration gradients, salt metathesis, and displacement by higher binding affinity head groups. ${ }^{64-70}$ Unfortunately, the same chemical principles that make 
these ligand exchange reactions viable also make them unstable in catalytically relevant conditions. Especially the strong acid conditions, typically $0.5 \mathrm{M}$ sulfuric acid, required for HER lead to the protonation and desorption of such ligands.

Thus, to explore the influence that ligand surface chemistry can have in altering the catalytic activity and stability of colloidal nanoparticles, we must first establish synthetic methods to create more robust nanoparticle-ligand bonds. One such method that has been explored in the literature is the covalent functionalization of surfaces by substituted aryldiazonium salts. Diazonium salts are a diverse class of organic reagents that have been shown to form covalent bonds with electronrich surfaces. ${ }^{71-76}$ Specifically, Saveant showed decades ago that these reagents could functionalize the surface of glassy carbon electrodes and that these functional groups were stable to physical abrasion and electrochemical cycling. ${ }^{77}$ More recently, diazonium reactivity has been explored with two-dimensional materials, namely $\mathrm{MoS}_{2}$ nanosheets. ${ }^{78,79}$ Miller and co-workers explored the effects of covalent surface functionalization on the catalytic activity of $\mathrm{MoS}_{2}$ for HER and found strong correlations between the functional group Hammett parameter, $\mathrm{MoS}_{2}$ electronic structure, and overpotential for HER. ${ }^{61}$ Though this chemistry has never been explored on colloidal heteroatomic nanoparticles, specifically transition metal phosphides, there is precedent for the approach being viable for P-functionalization based on studies on phosphorene. ${ }^{78,80}$

Herein, we explore the synthetic conditions required to make a well-defined covalent ligand shell on the surface of colloidally synthesized $\mathrm{Ni}_{2} \mathrm{P}$ nanocrystals. The effects of substituted aryl ligands on electronic structure, catalytic activity, and stability are presented using a combination of spectroscopic, electrochemical measurements, and density functional theory (DFT) calculations. Finally, synthetic challenges and design principles for future development of colloidal nanocrystal electrocatalysts are presented.

\section{Experimental Section}

2-propanol (anhydrous, 99.5\%), triethyloxonium tetrafluoroborate ( $\geq 97.0 \%$, Meerwein's reagent), acetonitrile (anhydrous, 99.8\%,), chloroform (anhydrous, $\geq 99$ ), 4-nitrobenzenediazonium tetra fluoroborate (97\%), 4-methoxybenezenediazonium tetrafluoroborate (98\%), 3,5dichlorobenzenediazonium tetrafluoroborate, and bi(cyclopentyldienyl)cobalt(II) were all purchase from Millipore-Sigma, stored in a nitrogen atmosphere glovebox, and used without further purification. Diazonium salts and Meerwein's reagent were stored at $-20{ }^{\circ} \mathrm{C}$ in a glovebox freezer. Tris-diethylaminophosphine (97\%, Millipore-Sigma) was stored in ambient conditions and used as received. Nickel (II) chloride (98\%, Millipore-Sigma) was dried at $100{ }^{\circ} \mathrm{C}$ under vacuum overnight before being stored in a nitrogen glovebox until use. Oleylamine (technical grade, 90\%), was dried over $\mathrm{CaH}_{2}$, distilled, and stored over $4 \AA$ sieves in a nitrogen glovebox.

\section{Synthetic Details.}

$\mathrm{Ni}_{2} \mathrm{P}$ nanocrystals were synthesized using air-free Schlenk techniques under an $\mathrm{N}_{2}$ atmosphere. Glassware, including a $100 \mathrm{~mL}$ three neck round bottom flask (RBF), two condensing columns (used without flowing water), a hose adapter, glass thermal well, Teflon magnetic stir bar, $100 \mathrm{~mL}$ Schlenk flash, canula needle, and 14/20 ground glass stopper were all dried in the oven at $160{ }^{\circ} \mathrm{C}$ overnight prior to use. The next day, the reaction vessel was assembled on top of a heating mantle and connected to the Schlenk line, evacuated, and refilled with dry $\mathrm{N}_{2}$. In an $\mathrm{N}_{2}$ glovebox, $944 \mathrm{mg}$ (7.2 mmol) of $\mathrm{NiCl}_{2}$ was measured. $\mathrm{NiCl}_{2}$ was quickly transferred out of the glovebox and added to the reaction vessel. The vessel was purged with high vacuum/ $\mathrm{N}_{2}$ flushes three times. $48 \mathrm{~mL}$ (146 mmol or $20 \mathrm{~mol}$ eq. relative to $\mathrm{Ni}$ ) of oleylamine was added to the reaction vessel under an 
$\mathrm{N}_{2}$ atmosphere through a rubber stopper. The mixture of $\mathrm{NiCl}_{2}$ and oleylamine was heated to 120 ${ }^{\circ} \mathrm{C}$ and vigorously stirred under vacuum for 60 min to remove trace $\mathrm{O}_{2} / \mathrm{H}_{2} \mathrm{O}$ and volatile organic contaminants. The vessel was wrapped in glass wool to promote uniform heating. The reaction temperature was measured by an internal probe, submerged in the reaction vessel through contact with a glass thermal well. After $60 \mathrm{~min}$, the reaction mixture was cooled to $\sim 50{ }^{\circ} \mathrm{C} .8 \mathrm{~mL}(28.8$ mmol or 4 mol eq. relative to $\mathrm{Ni}$ ) tris-diethylaminophosphine was injected through the rubber stopper. The reaction vessel was then heated to $250{ }^{\circ} \mathrm{C}$ at a ramp rate of roughly $10{ }^{\circ} \mathrm{C} \mathrm{min}{ }^{-1}$ and held for $60 \mathrm{~min}$. After about $12 \mathrm{~min}$, at $178{ }^{\circ} \mathrm{C}$, a red hue was observed. The solution quickly became black as the temperature climbed above $200{ }^{\circ} \mathrm{C}$. At this point vapors were observed in the vessel. After 1 hour at $250{ }^{\circ} \mathrm{C}$ the vessel was lifted from the heating mantle and the glass wool was removed, allowing the solution to cool.

As the solution cooled, an oven dried Schlenk flask was attached to the Schlenk line and purged with three flushes of vacuum/ $\mathrm{N}_{2}$ and finally held under $\mathrm{N}_{2}$ flow. Once the reaction mixture reached $\sim 80{ }^{\circ} \mathrm{C}$ it was transferred to the Schlenk flash via canula. The Schlenk flask was then sealed with a greased ground glass stopper which was fastened with electrical tape and brought into the glovebox. The reaction mixture was separated into 6 individual $45 \mathrm{~mL}$ Falcon tubes, diluted with $2 \mathrm{x}$ volume of 2-propanol, and centrifuged at $7.83 \mathrm{kRPM}$ for $15 \mathrm{~min}$. This was repeated until the supernatant for all tubes was clear. The nanocrystals were redispersed in minimal pentane and diluted with 10x volume of 2-propanol before centrifugation at $7.83 \mathrm{kRPM}$ for $15 \mathrm{~min}$. This process was repeated three times, and then another three times using a toluene solvent/acetonitrile antisolvent (1:10) mixture. Finally, the cleaned product was dissolved in minimal chloroform and transferred to a tared $20 \mathrm{~mL}$ scintillation vial. Once the solvent was removed by vacuum the product appeared as a free-flowing black powder. $842 \mathrm{mg}$ of $\mathrm{Ni}_{2} \mathrm{P}$ was recovered.

The treatment of $\mathrm{Ni}_{2} \mathrm{P}$ with Meerwein's salt was carried out in a $250 \mathrm{~mL}$ round bottom flask that was dried a $160{ }^{\circ} \mathrm{C}$ overnight before use. $100 \mathrm{mg}$ of $\mathrm{Ni}_{2} \mathrm{P}$ was dissolved in $40 \mathrm{~mL}$ of toluene and $1.425 \mathrm{~g}$ of Meerwein's salt was dissolved in $50 \mathrm{~mL}$ of acetonitrile $(0.1 \mathrm{M}$ and $50 \mathrm{~mol}$ eq. relative to oleylamine, assuming it accounts for $20 \%$ of the $\mathrm{Ni}_{2} \mathrm{P}$ sample mass). These solutions were mixed and stirred vigorously for a few minutes to make a homogeneous dark black solution, then left still overnight. The next day a black precipitate was observed at the bottom of the flask. The clear solution was poured off, and the black powder was collected by addition of minimal fresh acetonitrile. Stripped particles, $\mathrm{Ni}_{2} \mathrm{Ps}$, were then moved to a $45 \mathrm{~mL}$ Falcon tube where another $10 \mathrm{~mL}$ of acetonitrile was added. The sample was centrifuged at $7.83 \mathrm{kRPM}$ for $15 \mathrm{~min}$, resulting in a clear supernatant that was decanted. This process was repeated twice more. Washed $\mathrm{Ni}_{2} \mathrm{Ps}$ was then transferred to a tared $20 \mathrm{~mL}$ scintillation vial as a solution in toluene, and then dried by vacuum.

Surface functionalization reactions were carried out in an $\mathrm{N}_{2}$ glovebox in $20 \mathrm{~mL}$ scintillation vials that were oven dried overnight at $160{ }^{\circ} \mathrm{C}$ before use. $40 \mathrm{mg}$ of $\mathrm{Ni}_{2} \mathrm{Ps}$ (when corrected for 15 mass\% oleylamine, this translates to $34 \mathrm{mg} \mathrm{Ni} 2 \mathrm{P}$ or $0.229 \mathrm{mmol} \mathrm{P}$ ) was dissolved in $10 \mathrm{~mL}$ of chloroform and added to a $20 \mathrm{~mL}$ scintillation vial with a Teflon stir bar and set to stir at $700 \mathrm{RPM}$. While stirring, in the cases that a chemical reductant was added, $0.115 \mathrm{mmol}(2 \mathrm{~mol}$ eq. to surface P, SI. S3) of the reducing agent (bi(cyclopentyldienyl)cobalt(II) or cobaltocene) was measured and added to the chloroform solution. Then, $0.115 \mathrm{mmol}$ ( $2 \mathrm{~mol}$ eq. to surface P, SI. S3) of the respective diazonium salt was weighed out and dissolved in $10 \mathrm{~mL}$ of acetonitrile. The diazonium solution was added to the vial of stirring $\mathrm{Ni}_{2} \mathrm{Ps}$ in a slow, dropwise manner. The effervescence of $\mathrm{N}_{2}$ in the form of small bubbles was observed after addition of the diazonium, signaling the initiation of radical formation. Solutions were allowed to stir while lightly capped for 48 hours. 
For purifying the functionalized nanocrystals, the solvent was first removed under vacuum. Once dried, $5 \mathrm{~mL}$ of clean acetonitrile was added to the residue and shaken to disperse. This solution was transferred to a $45 \mathrm{~mL}$ Falcon tube and centrifuged at $5 \mathrm{kRPM}$ for $10 \mathrm{~min}$. The supernatant, which had a faint yellow color, was either discarded or collected for analysis. Washing and centrifugation using fresh acetonitrile was repeated 3 more times. Samples were then dissolved in minimal chloroform, transferred to tared $20 \mathrm{~mL}$ scintillation vials, and pumped down to a fine, free flowing, black powder.

\section{Characterization.}

Powder X-ray diffraction (XRD) data was collected on a Bruker Microfocus instrument. Powder XRD samples were prepared by drop-casting a solution of $\mathrm{Ni}_{2} \mathrm{P}$ onto silicon single crystal wafers. Diffuse reflectance infrared Fourier transform spectroscopy (DRIFTS) was collected on a Bruker Alpha IR instrument. Samples were prepared by grinding the $\mathrm{Ni}_{2} \mathrm{P}$ together with FT-IR grade potassium bromide $(\mathrm{KBr},>99 \%$; Alfa Aesar) using an agate mortar and pestle. Background measurements were taken with a golden puck. ${ }^{1} \mathrm{H}$ and ${ }^{31} \mathrm{P}$ spectra were recorded on Bruker AV300 and Bruker AV500 spectrometers, respectively. Deuterated chloroform $\left(\mathrm{CDCl}_{3}\right)$ and acetonitrile $\left(\mathrm{CD}_{3} \mathrm{CN}\right)$ were purchased from Cambridge isotope labs and dried over calcium hydride, vacuumtransferred, and stored over $4 \AA$ sieves in a $\mathrm{N}_{2}$ glovebox. $\mathrm{CDCl}_{3}$ contained a $1 \%$ internal standard of tetramethyl silane. ${ }^{31} \mathrm{P}$ spectra were corrected to an $85 \%$ phosphoric acid standard in water. Xray photoelectron spectroscopy (XPS) was conducted on a Kratos AXIS Ultra DLD. Pass energy for survey spectra (to calculate composition) was $150 \mathrm{eV}$. Data point spacing was $1.0 \mathrm{eV}$ per step for survey spectra, and $0.4 \mathrm{eV}$ per step for detailed spectra. Pass energy for high-resolution spectra was $50 \mathrm{eV}$, with a data point spacing of $0.065 \mathrm{eV}$. All spectra were calibrated to align carbon peak intensities at $284.8 \mathrm{eV}$. Ultraviolet photoelectron spectroscopy (UPS) was also conducted on a Kratos AXIS Ultra DLD. The UV source was a He(I) lamp with photon energy of $21.2 \mathrm{eV}$. Pass energy was $5 \mathrm{eV}$ with a step size of $0.01 \mathrm{eV}$. Aperture (spot) size was $100 \mu \mathrm{m}$. Take off angle was 0 degrees, and the stage was biased by a $9 \mathrm{~V}$ battery while collecting data. All spectra were corrected to a sputter cleaned gold sample's fermi edge at $0 \mathrm{eV}$. Transmission electron microscopy (TEM) images were collected on an FEI Tecnai G2 F20 microscope. Samples were drop cast from toluene onto lacey carbon grids and vacuum dried overnight. Scanning electron microscopy (SEM) images were collected on a ThermoFisher Scientific Apreo Variable Pressure SEM with integrated energy dispersive x-ray spectrometer (EDS) from Oxford. Elemental quantification was carried out using a Perkin Elmer Optima 8300 Inductively Coupled Plasma-Optical Emission Spectrophotometer (ICP-OES). Thermogravimetric Analysis (TGA) measurements experiments were carried out using a TA Instruments TGA Q5000. $\sim 10 \mathrm{mg}$ of material was loaded onto an aluminum pan at ambient conditions, then raised to $500{ }^{\circ} \mathrm{C}$ at a ramp rate of $10{ }^{\circ} \mathrm{C} / \mathrm{min}$ under $\mathrm{N}_{2}$ with a flow rate of $25 \mathrm{~mL} / \mathrm{min}$. All electrochemical measurements were performed with a Gamry Interface 1000. Measurements were conducted under $\mathrm{Ar}$ flow in $0.5 \mathrm{M} \mathrm{H}_{2} \mathrm{SO}_{4}$ with $18 \mathrm{M} \Omega$ Millipore water in a custom four-neck cell fitted with a graphite rod counter electrode separated in a fritted compartment, a $\mathrm{Ag} / \mathrm{Ag}_{2} \mathrm{SO}_{4}$ reference electrode separated by a Vycor frit, and a glassy carbon working electrode. A desired volume of a known mass\% solution was drop cast onto the glassy carbon electrode to disperse the catalyst. Glassy carbon electrodes were consecutively polished with 5,1 , and $0.05 \mu \mathrm{m}$ alumina paste and sonicated/washed with $18 \mathrm{M} \Omega$ Millipore water prior to use.

\section{DFT Calculations.}


Spin-polarized DFT calculations were carried out using the Quantum ESPRESSO ${ }^{81}$ package (v6.5). Exchange-correlation effects are described using the Perdew-Burke-Ernzerhof (PBE) functional ${ }^{82}$ along with the semi-empirical Grimme's D2 van der Waals (vdW) corrections. ${ }^{83}$ The bonding environment of each system is described under the projector augmented wave method $(\mathrm{PAW})^{84}$. The expansion of valence wave functions has been accounted for with a plane wave cutoff energy of $680 \mathrm{eV}$. All simulations used a $3 \times 3 \times 1 \Gamma$-centered Monkhorst-Pack ${ }^{85}$ k-point integration of the Brillouin zone. Surface reactions are modelled using an 8-layer periodically repeated hexagonal supercell $(11.8 \AA \times 11.8 \AA \times 41.6 \AA)$ belonging to the $\mathrm{P} \overline{62} \mathrm{~m}$ space group to model the coverage effect of surface adsorbates. A $25 \AA$ vacuum space was employed to prevent spurious interactions between the periodically repeated images. Atomic coordinates were relaxed using the Broyden-Fletcher-Goldfarb-Shanno ${ }^{86}$ (BFGS) algorithm until the Hellmann-Feynman forces on all relaxed atoms fell below $0.05 \mathrm{eV} / \AA$. For geometry optimization the electron occupancies were determined by the Gaussian smearing method with a smearing value of $0.07 \mathrm{eV}$, while for accurate total energy and partial density of states analysis we adopt the optimized tetrahedron method. ${ }^{87}$ Due to the relatively large system sizes in this study (144-204 atoms), only the top two layers are relaxed, with the bottom 6 layers remaining fixed to that of the bulk values. Preliminary analysis indicated that $\mathrm{C}_{6} \mathrm{H}_{4} \mathrm{NO}_{2}$ preferentially binds to the $\mathrm{P}$ top site with high coordination Ni and P sites being disfavored in terms of total energy (See SI for more details). The $\mathrm{Ni}_{2} \mathrm{P}(0001)$ slab with the $\mathrm{Ni}_{3} \mathrm{P}_{2}$ surface termination was chosen for three main reasons: (i) it closely resembles the experimental synthesis which has an observed stoichiometry of $\left(\mathrm{Ni}_{1.78} \mathrm{P}\right)$, (ii) it is known to be stable under the electrochemical conditions relevant in this study ${ }^{41}$ and (iii) it provides a point of reference with the current published literature.

\section{Results and Discussion}

Nickel phosphide synthesis and ligand stripping.

Nickel phosphide nanocrystals were prepared using a modified literature procedure. ${ }^{20}$ Briefly, nickel(II) chloride was mixed with tris(diethylamino)phosphine in neat oleylamine. After heating to $250{ }^{\circ} \mathrm{C}$ for $60 \mathrm{~min}$, phase pure $\mathrm{Ni}_{2} \mathrm{P}$ nanocrystals with an average diameter of $5 \mathrm{~nm}$ (Fig. 1A) were recovered from a viscous black reaction mixture after successive rounds of precipitation, centrifugation at $7830 \mathrm{RPM}$, and redissolution with isopropanol/pentane followed by toluene/acetonitrile. TEM images reveal an approximately spherical morphology with an average diameter of $5 \mathrm{~nm} \pm 0.9 \mathrm{~nm}$ (Fig. 1A). The powder XRD pattern is in agreement with the reported reference powder pattern (PDF 01-074-1385). (Fig. 1B). Scherrer analysis reflects an average crystalline domain size of $5.18 \mathrm{~nm}$, consistent with the TEM analysis. ICP-OES reveals a composition that is phosphorous rich, with an average stoichiometry of $\mathrm{Ni}_{1.78} \mathrm{P}$. This is a notable departure from the cation rich nature of more ionic nanomaterials synthesized with aminophosphine precursors, namely InP ${ }^{88,89}$ We believe this composition is reflective of the inorganic nanocrystal core composition since there was no trace of excess molecular precursor or phosphonium salt byproduct found by NMR or FTIR spectroscopy of the purified end material (SI Fig. S1 and S4a).

Analysis by diffuse reflectance infrared Fourier transform spectroscopy (DRIFTS) and ${ }^{1} \mathrm{H}$ NMR spectroscopy supports a ligand shell composed of oleylamine (SI Fig. S1). The IR spectra showed signatures of long-chain aliphatic hydrocarbons at 1,450,2,850, and 2,920 $\mathrm{cm}^{-1}$, as well as the expected alkene resonances at 1,600 and 3,000 $\mathrm{cm}^{-1} .{ }^{90,91}{ }^{1} \mathrm{H}$ NMR spectra show resonances associated with aliphatic protons in the range of $1-2 \mathrm{ppm}$ and the alkene protons at $5.3 \mathrm{ppm} .{ }^{92}$ Further, the broadness of these peaks is indicative of the long relaxation times expected for 
molecules bound to a nanoparticle surface ${ }^{65,93}$ Though previous reports of nanocrystals prepared using combinations of metal halide salts and aminophosphine precursors show evidence of a mixed ligand shell comprised of both amine and chloride ions, no evidence of $\mathrm{Cl}$ atoms are observed in the XPS survey spectrum (SI Fig. S2a) ${ }^{89}$ Further, thermogravimetric analysis suggests that the surface ligands compose $19 \%$ of the sample by mass (SI Fig. S3). The first derivative of mass loss for this sample shows a peak at $\sim 350{ }^{\circ} \mathrm{C}$, matching closely with the boiling point of neat oleylamine $\left(365^{\circ} \mathrm{C}\right)$.

A)

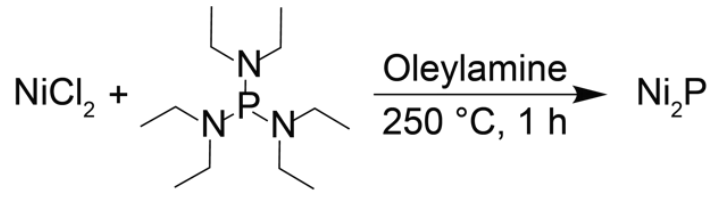

C)
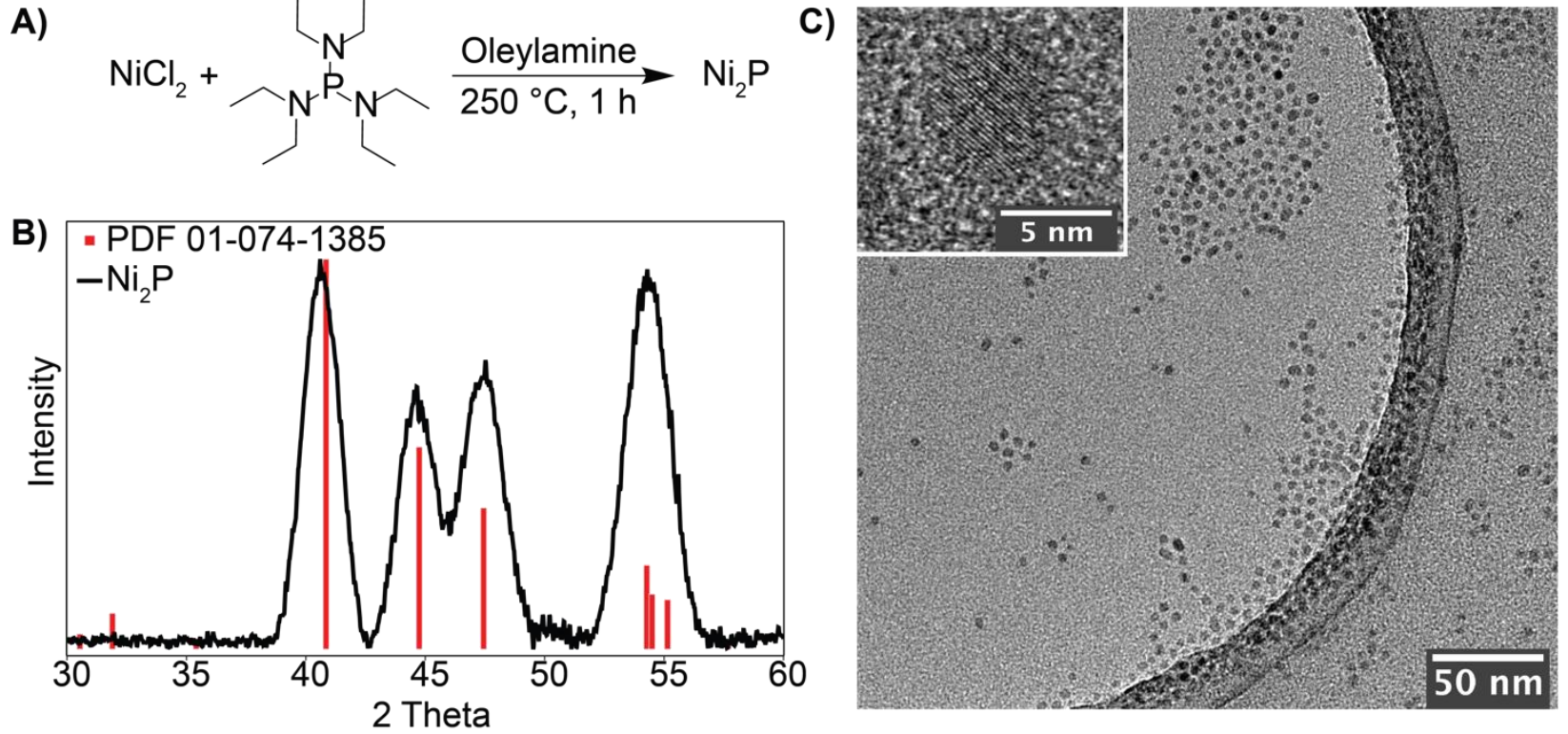

Figure 1. A) General reaction scheme for the synthesis of $\mathrm{Ni}_{2} \mathrm{P}$ nanocrystals with aminophosphines. B) XRD of $\mathrm{Ni}_{2} \mathrm{P}$ nanocrystals references against powder pattern (PDF 01-0741385). C) TEM of $\mathrm{Ni}_{2} \mathrm{P}$ product, showing $5 \pm 0.9 \mathrm{~nm}$ spherical nanocrystals. Inset shows a single nanocrystal with a $5 \mathrm{~nm}$ scale bar for reference. Full image reflects a larger sample set of nanocrystals all reflecting similar sizes and morphologies with a $50 \mathrm{~nm}$ scale bar for reference.

High resolution XPS analysis of the Ni and P $2 p$ electron binding energies are consistent with a covalent metal-phosphide material. ${ }^{38,28}$ The P 2 p spectrum (SI Fig S2c) shows a spin-orbit doublet with the center of the parent peak located at $129 \mathrm{eV}$ consistent with $\mathrm{P}$ in the 0 oxidation state and representing $72 \%$ of the total $\mathrm{P}$ in the sample. A higher energy shoulder centered at 131.5 $\mathrm{eV}$ was fit to a broad doublet peak, representing what is likely multiple ill-defined higher oxidation state $\mathrm{P}$ components within the material. This species comes at a slightly lower binding energy than is expected of phosphate salts $(132.5 \mathrm{eV})$, which leads us to believe it is likely a partially oxidized surface site. ${ }^{94}$ Similarly, the Ni $2 \mathrm{p}$ spectrum shows an unusual Ni binding energy of $853 \mathrm{eV}$, which lies in-between that of $\mathrm{Ni}^{0}$ and $\mathrm{Ni}^{2+}\left(852 \mathrm{eV}\right.$ and $854 \mathrm{eV}$, respectively). ${ }^{95}$ This result is indicative of the relatively high amount of electron density that remains on the Ni sites within the phosphide lattice. The 2 $\mathrm{p}_{3 / 2}$ environment can be deconvoluted into two contributions. The first being the metal-phosphide species centered at $853 \mathrm{eV}$ which represents $72 \%$ of the sample. The second, centered at $853.5 \mathrm{eV}$, accounts for $28 \%$ of the sample and is likely the slightly reduced surface oxide, similar to that observed in the $\mathrm{P} 2 \mathrm{p}$ spectrum.

Prior to covalent functionalization of $\mathrm{Ni}_{2} \mathrm{P}$, the concentration of native oleylamine ligands was reduced via alkylation using Meerwein's salt. Alkylation is a widely used method for the removal 
of common ligands including oleylamine, oleate, and phosphate species. ${ }^{96,97}$ Typically, ligand stripping procedures for colloidally dispersed nanoparticles consist of preparing a biphasic solution where the non-polar phase contains the native nanocrystals dissolved in hexanes and the polar phase is a solution of Meerwein's salt in acetonitrile or DMF. Vortex mixing leads to rapid precipitation of the nanocrystals from solution. The recovered nanocrystals can be washed to remove residual aliphatic ligands before the particles are able to be resuspended by solvents with weak binding affinities such as DMF. In the most successful examples, complete loss of aliphatic C-H vibrations in FTIR spectra coupled with elemental analysis showing a lower concentration of $\mathrm{C}$ atoms are observed. ${ }^{96,98}$ Interestingly, this method of ligand stripping was not as efficient in the case of the oleylamine-ligated $\mathrm{Ni}_{2} \mathrm{P}$ nanocrystals studied here. Following synthesis and purification, the $\mathrm{Ni}_{2} \mathrm{P}$ nanocrystals showed only sparing solubility in hexanes, limiting our ability to directly use the procedure as previously reported. Additionally, vortex mixing the biphasic solution resulted in formation of a highly viscous gel. Ultimately, the ligand exchange was accomplished using a homogenous solution of the nanocrystals $(5 \mathrm{mg} / \mathrm{mL})$ in toluene with a large excess of Meerwein's salt (50 mol equiv. vs oleylamine) in acetonitrile. This solution was mixed vigorously for several minutes in a round bottom flask and left to sit overnight, resulting in the formation of a black precipitate. The supernatant was decanted, and the resulting nanocrystal powder was dissolved in toluene and subjected to repeated rounds of precipitation and redissolution from acetonitrile.

The Meerwein's salt-treated nanocrystals were characterized by TGA, IR, and NMR spectroscopy (SI Fig. S3-4). These data suggest that roughly 30\% of the initial oleylamine surface ligands were removed. However, oleylamine still accounted for $15 \%$ of the sample by mass, and signature spectroscopic features were still observed in the DRIFTS and ${ }^{1} \mathrm{H}$ NMR spectra. We do note that changes in the ${ }^{31} \mathrm{P}$ NMR spectra suggest that alkylation is an effective method to remove the surface phosphate species that formed from ambient oxidation. Successive alkylation treatments had limited added effect and we have not managed to realize complete ligand removal in this system (SI Fig. S3), suggesting that the starting nanocrystals have a range of amine binding energies and that only the weakest bound subset can be removed using this method. This result stands in contrast to earlier examples of Meerwein's salt treatment of nanocrystals for ligand stripping, a fact that may be attributable to differences in the covalent bonding character of the $\mathrm{Ni}_{2} \mathrm{P}$. Early examples from Helms and co-workers showcased the method's efficacy on highly ionic materials such as $\mathrm{CdSe}, \mathrm{TiO}_{2}$, and ITO. ${ }^{96}$ Later studies, however, have shown less consistent results. In a study from Suntivich et al. TGA suggested that alkylation did not remove all oleate ligands from the surface of $\mathrm{Mn}_{3} \mathrm{O}_{4}$ particles, despite the ionic nature of that lattice. ${ }^{97}$ In contrast, a recent paper from Buonsanti et al. showed complete removal of native phosphate and trialkylamine ligands from the surface of $\mathrm{Cu}^{0}$ nanospheres. ${ }^{98}$

\section{Covalent functionalization.}

To a chloroform solution of Meerwein's salt-treated $\mathrm{Ni}_{2} \mathrm{P}$ nanocrystals $\left(\mathrm{Ni}_{2} \mathrm{Ps}\right)$ a solution of diazonium salt in acetonitrile was added dropwise (Fig. 2A). A spontaneous reaction was indicated by effervescence as $\mathrm{N}_{2}$ gas was released. In some instances, a toluene solution of cobaltocene was added dropwise prior to addition of diazonium. In either case, the solution was capped loosely and left to stir for 48 hours. The functionalized nanocrystals were purified by first removing the solvent from the crude mixture under vacuum. Once the sample was dry, the powder was dispersed in acetonitrile by vigorous shaking or sonication, followed by multiple rounds of precipitation via centrifugation, removal of supernatant, and redissolution in acetonitrile to remove excess 
diazonium salt. Subsequent washes with isopropanol and/or pentane were performed to remove any aliphatic reaction co-products. After multiple washes, the particles remained highly soluble in chloroform and/or toluene. Solvent removal under vacuum resulted in a free-flowing black powder.

We note that during initial washing steps the supernatant was observed to have a slightly yellow hue. Upon further investigation, we determined this side product was likely the result of reactivity between the diazonium ions and oleylamine. In fact, diazonium salts have been previously documented to act as ligand stripping reagents for colloidal quantum dots. ${ }^{99}$ Though the authors did not speak much to the mechanism, they showed the loss of prominent aliphatic vibrations after treatment and the growth of a $\mathrm{BF}_{4}$ signal - suggesting some sort of ion exchange reaction. We propose that the mechanism is likely much more complicated due to the complex reactivity of highly electrophilic diazonium ions and nucleophilic primary amines. ${ }^{100}{ }^{1} \mathrm{H}$ NMR spectra of oleylamine, 4-nitrobenezene diazonium tetrafluoroborate, and an equimolar mixture of the two can be found in the supporting information (Fig. S6). Here we can observe the degradation of the clean aryl doublets $(8.8$ and $8.6 \mathrm{ppm})$ found in the parent diazonium into a convolution of multiple aryl species that are more electron rich, as suggested by their downfield shift (spanning from 7.6 to $8.3 \mathrm{ppm}$ ). We also observe that the reaction continues to evolve over the course of 2 days, by which point the original aryl resonances are no longer observed. We compare this control sample to the supernatant from our $\mathrm{Ni}_{2} \mathrm{P}_{-} \mathrm{C}_{6} \mathrm{H}_{4} \mathrm{NO}_{2}$ sample and observe many similar features, including the convoluted aryl signals and overwhelming aliphatic protons from the oleylamine.

A survey of early work studying the reactivity of diazonium ions lays out multiple paths of reactivity between these species, including nucleophilic attack at the beta-N, substitution at the C2 and C-4 positions, heterolytic dediazoniations, and homomolecular coupling. ${ }^{100}$ This wide array of possible products is even further muddied by the influence of catalytic nanoparticles that provide competing bonding affinities, reducing equivalents, and catalytically active surfaces that can alter the reaction landscape. Further, the comparison of reaction supernatant and control sample suggest the reaction products are different, or at least are formed in very different ratios. However, none of these spectral features are observed in the purified products (Fig. S7) suggesting that these species can be completely removed from the system by thorough washing and do not result in persistent contamination.
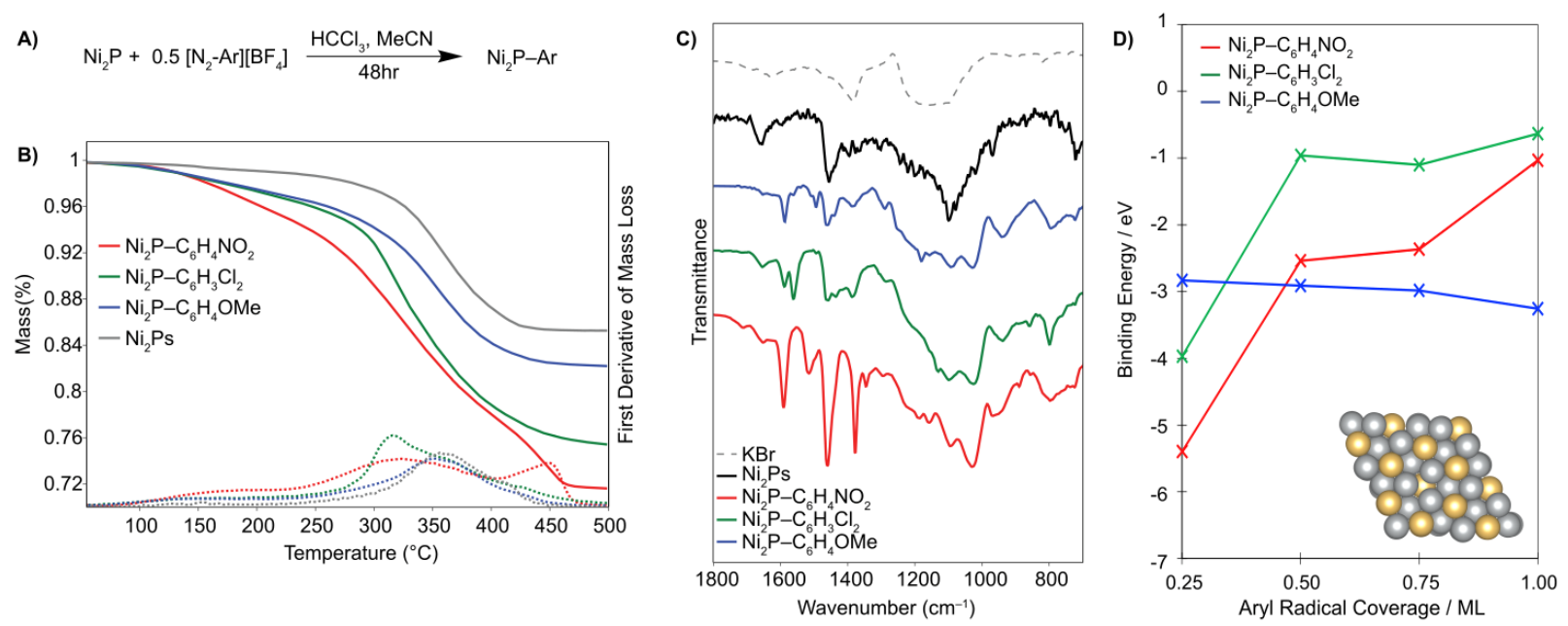
Figure 2. A) General reaction scheme for spontaneous functionalization of Meerwein's treated $\mathrm{Ni}_{2} \mathrm{P}$ nanocrystals with substituted aryldiazonium salts. B) DRIFTS spectrum of nanocrystals dispersed in $\mathrm{KBr}$ matrix. Diagnostic peaks are discussed in the main text. C) TGA trace of nanocrystals heated to $500{ }^{\circ} \mathrm{C}$ at $10{ }^{\circ} \mathrm{C} / \mathrm{min}$. Solid lines represent percent mass loss relative to the initial sample mass. Dotted lines show the first derivative of the mass loss curve, describing temperature ranges with sharper mass losses. D) DFT calculated aryl radical binding energy as a function of surface coverage; the insert depicts the bare $\mathrm{Ni}_{3} \mathrm{P}_{2}(0001)$ surface.

Evidence of spontaneous functionalization of the $\mathrm{Ni}_{2} \mathrm{P}$ nanocrystals was provided by TGA and DRIFTS of the purified and dried nanocrystal powders. The DRIFTS spectra reflected a mixed ligand shell, composed of remnant oleylamine and substituted aryl groups. Importantly, when comparing the spectra of functionalized nanocrystals to the parent diazonium salt (Fig. $2 \mathrm{C}$ and S8-10) we observed the absence of the N-N triple bond at $\sim 2200 \mathrm{~cm}^{-1}$. This is evidence that the nanocrystals were able to reduce the parent diazonium, cleaving the $\mathrm{N}_{2}$-Ar bond. Further, this implies that the new spectral features observed in the fingerprint region are from surface bound functional groups rather than remnant/physiosorbed diazonium salt. Closer analysis of the features found in the fingerprint region show key vibrational modes associated with the specific functional groups expected in each sample, despite overlapping signal from oleylamine at $\sim 1450 \mathrm{~cm}^{-1} .{ }^{90}$ In the $\mathrm{Ni}_{2} \mathrm{P}-\mathrm{C}_{6} \mathrm{H}_{4} \mathrm{OMe}$ sample, the Ar-O stretch at $1291 \mathrm{~cm}^{-1}$ as well as the $\mathrm{O}-\mathrm{CH}_{3}$ rocking mode at $1180 \mathrm{~cm}^{-1}$ were observed. An additional peak at $1493 \mathrm{~cm}^{-1}$ was attributed to aryl ring deformations induced by electron donating groups, thus only being observed in this sample. The peak observed from $1586-1590 \mathrm{~cm}^{-1}$ in each sample is another characteristic aryl ring deformation attributed to $\mathrm{C}=\mathrm{C}-\mathrm{C}$ stretches. ${ }^{101}$ Characteristic symmetric and asymmetric $\mathrm{N}-\mathrm{O}$ stretches were observed in the $\mathrm{Ni}_{2} \mathrm{P}-\mathrm{C}_{6} \mathrm{H}_{4} \mathrm{NO}_{2}$ sample at 1513 and $1346 \mathrm{~cm}^{-1}$, respectively. The in and out of plane NO scissor modes were difficult to resolve but could be observed at 856 and $724 \mathrm{~cm}^{-1}$, respectively. Interestingly these values were shifted to slightly lower frequencies than those of the parent diazonium salt, implying that the nanoparticle surface is acting as an electron donor, weakening the $\mathrm{N}-\mathrm{O}$ bond. ${ }^{102}$ We note that all of the observed vibrational frequencies in our sample are in agreement with those previously reported in the functionalization of $\mathrm{MoS}_{2}$ by aryl diazonium salts, implying the distortion of bond energies via electronic effects from the underlying material is a common theme. ${ }^{61,103}$

TGA data (Fig. 2B) confirm a higher degree of functionalization with increasing Hammett parameter of the aryl substituent $\left(\mathrm{NO}_{2}>\mathrm{Cl}_{2}>\mathrm{OMe}\right)$, and hence increasing reactivity of the diazonium salt. This is consistent with previous observations in the literature, with the note that many papers use different methods of reporting the degree of functionalization depending on the system under investigation. For example, in studies of electrografting diazoniums onto glassy carbon surfaces authors report the magnitude of reductive current as a measure of functionalization yield. ${ }^{72,77,104,105}$ Another common method is reporting atomic ratios determined by XPS or S-Mo:S$\mathrm{C}$ ratios in the case of $\mathrm{MoS}_{2}$ functionalization. ${ }^{61,79,106}$ Though many of these studies supplement their findings with TGA and find the same result. We note, however, that the TGA data collected here is convoluted by the persistence of oleylamine in our sample, precluding direct correlation of the absolute mass loss values to covalently bound functional groups. In any case, we estimate the surface coverage of each sample with the assumption that $15 \%$ mass loss from oleylamine is constant across all samples, and that any further mass loss is from covalently bound surface functional groups. With these assumptions, we observed an upper bound for functionalization of $14 \%$ by mass for the nitrophenyl group. Assuming a $100 \mathrm{mg}$ sample, this translates to $14 \mathrm{mg}$, or 
$114 \mu \mathrm{mol}$ of nitrobenzene equivalents. In the same sample, $71 \mathrm{mg}$ of $\mathrm{Ni}_{2} \mathrm{P}$ would translate to 119 $\mu \mathrm{mol}$ of surface $\mathrm{P}$ sites, translating to $96 \%$ surface $\mathrm{P}$ sites being functionalized. Following this logic, methoxybenzene and 3,5-dichlorobenzene samples translate to 27 and $68 \mu \mathrm{mol}$, or $20 \%$ and 54\% surface P-Ar sites, respectively (example calculation in SI). All functionalization densities were below $100 \%$ saturation and were less than the molar equivalents of parent diazonium added to the reaction mixture.

DFT calculations support the idea that adsorption of $\mathrm{C}_{6} \mathrm{H}_{4}-\mathrm{NO}_{2}, \mathrm{C}_{6} \mathrm{H}_{3}-\mathrm{Cl}_{2}$ and $\mathrm{C}_{6} \mathrm{H}_{4}-\mathrm{OCH}_{3}$ is spontaneous (Fig. 2D). Here, adsorbate-adsorbate and adsorbate-substrate interactions dictate the binding energy trends, with the electron donating/withdrawing character playing a key role. For instance, the adsorption energy of the electron withdrawing radicals $\left(\mathrm{C}_{6} \mathrm{H}_{3}-\mathrm{Cl}_{2}\right.$ and $\left.\mathrm{C}_{6} \mathrm{H}_{4}-\mathrm{NO}_{2}\right)$ become less favorable as we increase the surface coverage. While the electron donating propensity of $\mathrm{C}_{6} \mathrm{H}_{4}-\mathrm{OMe}$ counteracts the effects of adsorbate-adsorbate interaction, highlighted by the near constant binding energy as a function of surface coverage. Similar trends in binding energy have been observed in the literature for black phosphorous ${ }^{107}$ and molybdenum disulfide ${ }^{108}$. It should be noted that these calculations do not include thermal effects, which could significantly destabilize the adsorption of diazonium salts under reaction conditions. Other factors, such as, the presence of excess oleylamine capping ligands is shown to further destabilize the adsorption energetics (Fig. S23). That said, the low coverage trend of these species is consistent with the observed experimental coverages: $96 \%\left(\mathrm{C}_{6} \mathrm{H}_{4}-\mathrm{NO}_{2}\right), 54 \%\left(\mathrm{C}_{6} \mathrm{H}_{3}-\mathrm{Cl}_{2}\right)$ and $20 \%\left(\mathrm{C}_{6} \mathrm{H}_{4}-\mathrm{OMe}\right)$.

To address the issue of unsaturated surface coverage, presumably due to the insufficient reducing potential or equivalents in the reaction mixture, the effect of adding a molecular reductant was investigated. This concept was recently validated by the Lewis group who showed that the functionalization density of $\mathrm{MoS}_{2}$ by alkyl iodide species could be increased by adding sacrificial reductants to the reaction mixture. ${ }^{109}$ This study demonstrated a strong correlation between the strength of the reductant and the coverage of functional groups, supporting our hypothesis that each surface functional group equivalent becomes increasingly difficult to generate and is strongly dependent on the thermodynamic driving force for both generating the radical species and facilitating surface-C bond formation.

We experimentally validated this hypothesis in our own system by adding equimolar concentrations of cobaltocene (relative to diazonium) to our reaction mixtures. The resulting differences between batches of $\mathrm{Ni}_{2} \mathrm{P}_{-} \mathrm{C}_{6} \mathrm{H}_{4} \mathrm{NO}_{2}$ is shown in Fig. 3. The TGA data show a clear increase in mass loss and suggest that the organic content has increased to roughly $40 \%$ of the total sample mass. Following our earlier estimate of surface coverage, this would reflect a large excess ( $225 \%$ of surface $\mathrm{P}$ sites) of nitrobenzene groups. This super saturation can be rationalized by the formation of hyperbranched nitrophenyl oligomers on the nanocrystal surface, as shown in Fig. 3D. Uncontrolled reactivity, or hyperbranching, is a common phenomenon observed in diazonium chemistry. It is well known that aryl radicals react readily with $\mathrm{sp}^{2}$ carbons, hence their usefulness in functionalizing graphitic carbons. As such, they are also able to react with the $\mathrm{sp}^{2}$ carbons present in surface arenes. This can lead to the formation of large polymeric films, sometimes on the order of tens of nanometers thick. ${ }^{110}$ 
A)

$$
\mathrm{Ni}_{2} \mathrm{P}+0.5\left[\mathrm{~N}_{2}-\mathrm{C}_{6} \mathrm{H}_{4} \mathrm{NO}_{2}\right]\left[\mathrm{BF}_{4}\right] \frac{0.5 \mathrm{CoCp}}{\underset{48 C l_{3}, \mathrm{MeCN}}{48 \mathrm{hr}}}-\mathrm{Ni}_{2} \mathrm{P}_{2}-\mathrm{C}_{6} \mathrm{H}_{4} \mathrm{NO}_{2}
$$

B)

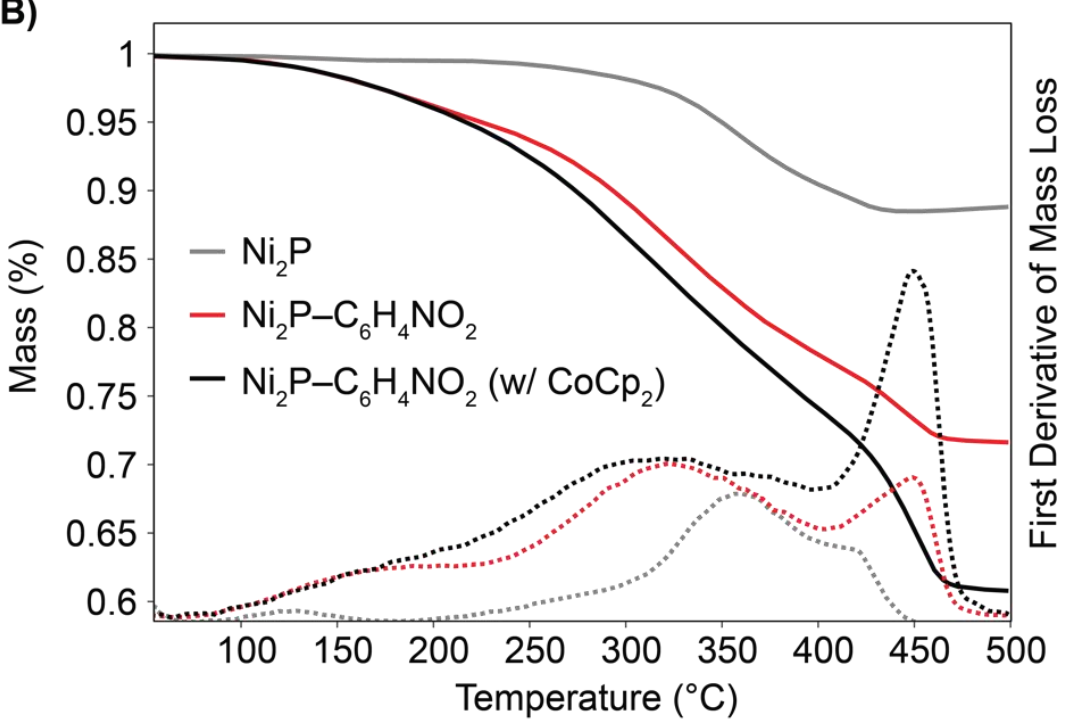

C)

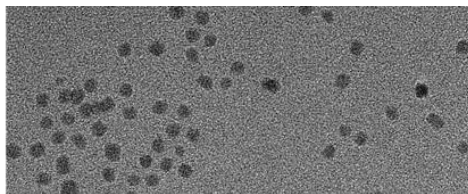

D)

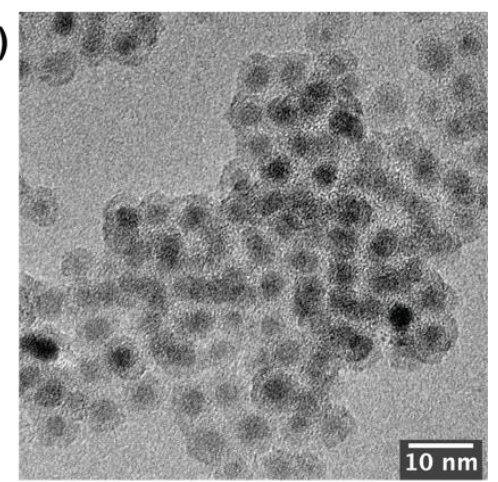

Figure 3. A) Reaction scheme showing functionalization of $\mathrm{Ni}_{2} \mathrm{P}$ using $\left[\mathrm{N}_{2}-\mathrm{C}_{6} \mathrm{H}_{4} \mathrm{NO}_{2}\right]\left[\mathrm{BF}_{4}\right]$ with the addition of cobaltocene as a sacrificial reductant. B) TGA of both samples, showing an increase in mass loss percent for samples made with added cobaltocene. Dotted lines show the first derivative of the mass loss percent line. C) TEM of $\mathrm{Ni}_{2} \mathrm{P}-\mathrm{C}_{6} \mathrm{H}_{4} \mathrm{NO}_{2}$ nanocrystals synthesized with no added reductant. Particles are still uniform and maintain their morphological integrity. Scale bar is $10 \mathrm{~nm}$. D) TEM of $\mathrm{Ni}_{2} \mathrm{P}-\mathrm{C}_{6} \mathrm{H}_{4} \mathrm{NO}_{2}$ synthesized with added cobaltocene. Particles show aggregation induced by the extensive aryl-multilayer film formation caused by aryl radical hyperbranching. Scale bar is $10 \mathrm{~nm}$.

\section{Effect of Functionalization on the Electronic Structure.}

The higher concentration of functional groups in the samples, derived from reactions in which a reductant was added, makes observation of functional groups with XPS easier. Spectra showing the presence of $\mathrm{Cl}$ and $\mathrm{NO}_{2}$ functional groups can be found in the supporting information (Fig. S13). What's more interesting, is the comparison of the $\mathrm{Ni}$ and $\mathrm{P} 2 \mathrm{p}$ high resolution scans between $\mathrm{Ni}_{2} \mathrm{P}-\mathrm{C}_{6} \mathrm{H}_{4} \mathrm{NO}_{2}$ samples made with and without added reductant (Fig. 4A and 4B). Rather than distinct peaks indicating the formation of a P-Ar or Ni-Ar bonds, we observe systematic shifts of the entire 'metal phosphide' signal. Although this result is surprising, it can be interpreted as the functionalization perturbing core-level electron density throughout the entire nanoparticle, which is reflected in the electronic density of states (DOS) inferred from the DFT calculations (Fig. S25). For the bare surface, the partial DOS plot shows that $\mathrm{Ni}_{2} \mathrm{P}$ exhibits metallic type conductivity with the states around the Fermi level consisting largely of Ni 3d-states with only a minor contribution from the overlapping P 3p-states. On adsorption of nitrobenzene groups, we observe a clear perturbation of these states and redistribution of electrons due to the electron withdrawing properties of the adsorbate. Despite our model only considering diazonium species adsorbed on the P-top sites, the change in total DOS can be attributed to a shift of both the P $3 p$-states and $\mathrm{Ni}$ $3 \mathrm{~d}$-states as-well-as a local increase in the P 3 -states. While we would typically expect this shift 
in electron density to be localized and correlated with the functionalized surface sites, we propose the high level of covalency, and the metallic character of these metal phosphide nanocrystals allow localized perturbations to be compensated for by the 'bulk' material. Further, the same trend is reflected in the work function of these materials. The work function is made more positive with higher degrees of functionalization, which can be interpreted as the highly polar nitrophenyl functional groups inducing a stronger surface dipole moment away from the crystal's core, in-turn requiring more energy to remove an electron from valence orbitals to vacuum.
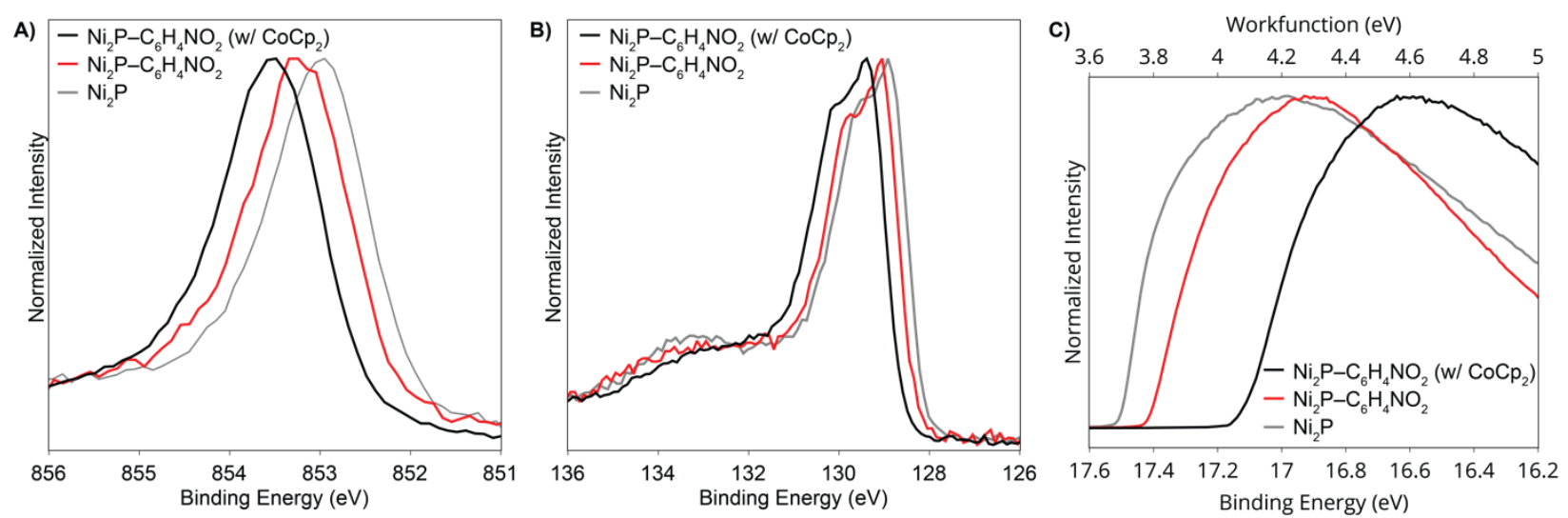

Figure 4. A) High resolution XPS spectra showing the gradual progression of the Ni 2p $3 / 2$ signal shifting to higher binding energies as the coverage of nitrobenzene groups increases (blue $>$ red). B) P 2p spectra of the same sample set, showing a similar systematic shift. C) UPS spectra of the same samples, showing the increased binding energy of the secondary electron cut off point, or work function. This illustrates increasing difficulty to eject an electron from the surface of the material as the coverage of nitrobenzene groups increases.

Although this phenomenon is unexpected when compared to studies of exfoliated $\mathrm{MoS}_{2}$ and black phosphorous nanosheets, it is reminiscent of the effects observed when doping metal phosphides with metals of varying electronegativity. ${ }^{111}$ In this study, Mar and coworkers observed a systematic shift in the $\mathrm{P}$ and $\mathrm{Ni} 2 \mathrm{p}_{3 / 2}$ binding energy over the range of $\sim 500 \mathrm{meV}$. The authors find that as more electropositive ions $(\mathrm{Co}<\mathrm{Fe}<\mathrm{Mn}<\mathrm{Cr})$ are doped into the lattice the $\mathrm{P} 2 \mathrm{p}_{3 / 2}$ binding energy systematically decreases. They rationalize this finding by considering a charge potential model to balance the inter and intraatomic effects of dopants on the P atoms in the lattice. ${ }^{112}$ Based on this model, intraatomic effects would lead to a decrease in binding energy due to increased nuclear screening and greater negative charge of $\mathrm{P}$ core electrons induced by their more electropositive environment. In contrast, interatomic effects would lead to a positive shift in binding energy due to the general increase in Madelung potential felt by each atom in the more electropositive lattice. We believe this rationale supports our interpretation of highly coupled covalent surface functional groups being able to perturb the 'bulk' electronic structure of the nanocrystal by inducing strong interatomic screening effects. The result of which is a systematic perturbation of the core-level binding energies in both $\mathrm{Ni}$ and $\mathrm{P}$ based on the degree of electron density that is donated or withdrawn by the surface functional group.

We further validate this hypothesis by showing systematic variation of core-level binding energies and NC work function across our Hammett series (Fig. 5). There is a clear linear trend observed in both $\mathrm{Ni}$ and $\mathrm{P} 2 \mathrm{p}$ high-resolution spectra where a larger Hammett parameter increases the core-level binding energy. This effect is most pronounced in the comparison of work functions 
across the Hammett series shown in Fig. 5C. To ensure this wasn't purely an electrostatic screening effect, we validated our hypothesis against a computational model where the same trend is observed (Fig. S24E).
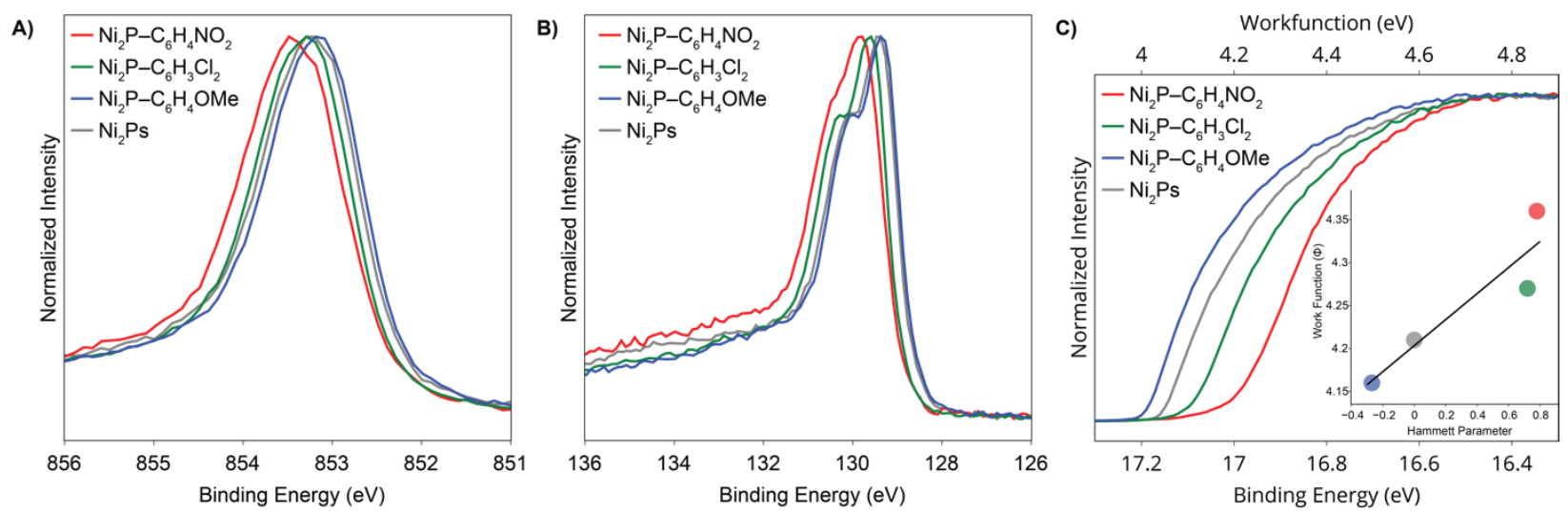

Figure 5. A) High resolution XPS spectra showing the shift of Ni $2 \mathrm{p}_{3 / 2}$ signal in response to the Hammett parameter of the surface functional group. B) P $2 p$ spectra of the same sample set, showing a similar systematic shift. C) UPS spectra of the same samples, showing the response of material work function to the Hammett parameter of surface functional group. Inlet shows the linear correlation between these descriptors.

\section{Electrocatalytic HER Activity.}

To test the effect that surface functionalization has on the electrocatalytic activity for HER of the $\mathrm{Ni}_{2} \mathrm{P}$ nanocrystals, $20 \mu \mathrm{L}$ of a $10 \mathrm{mg} / \mathrm{mL}$ solution of $\mathrm{Ni}_{2} \mathrm{P}$ nanocrystals in toluene were drop casted onto a $1.5 \mathrm{~mm}$ diameter glassy carbon electrode. Samples were prepared in a glovebox and transferred to a vessel of $0.5 \mathrm{M} \mathrm{H}_{2} \mathrm{SO}_{4}$ that was sparged with argon and held under a constant argon flow. Here we report the current density normalized by the geometric surface area of the glassy carbon electrode due to inconsistencies in estimating the electrochemically active surface area of the nanocrystal films (Figs. S14-S18). ${ }^{113}$ Metal phosphides are known to have exceptionally high specific capacitance values due to pseudocapacitive effects, which typically leads to over estimation of electrochemically active area. ${ }^{31,114,115}$ These pseudocapacitive properties would be affected by alteration of surface charge induced by the surface functional groups. This added complication makes it difficult to uniformly attribute "capacitive" current to strictly ion adsorption on a metallic surface, which is the base assumption in the estimation of electrochemically active surface area. ${ }^{116}$ This is reflected in our inability to find a potential region that reflects a purely capacitive response characterized by a square-wave with equal anodic and cathodic current. Instead, we observe variable cathodic current around the open-circuit potential with added resistive losses, potentially due to mass-transport limitation though the nanocrystal film (Figs. S15-S18). Though this may preclude us from reporting the true intrinsic activity of our catalysts, we can still compare relative activity within the series.

The catalytic activity results are summarized in Fig. 6 and reflect multiple key results. First, we observe over the course of 24 hours and 75 polarization cycles that the catalytic activity does not track the Hammett parameter, instead $\mathrm{Ni}_{2} \mathrm{P}-\mathrm{C}_{6} \mathrm{H}_{4} \mathrm{OMe}$ is the most active catalyst followed by $\mathrm{Ni}_{2} \mathrm{P}-\mathrm{C}_{6} \mathrm{H}_{4} \mathrm{NO}_{2}$ and then $\mathrm{Ni}_{2} \mathrm{P}-\mathrm{C}_{6} \mathrm{H}_{3} \mathrm{Cl}_{2}$ being the least active. This contrasts with previous studies by Miller and co-workers on covalently functionalized $\mathrm{MoS}_{2}$, where they found that catalytic activity was correlated with the functional group's Hammett parameter, although the nitrobenzene 
functionalized sample was not measured in that instance. ${ }^{61}$ Comparisons between these systems brings us to our second key result, which is improved catalytic activity in all functionalized samples when compared to $\mathrm{Ni}_{2} \mathrm{P}$ in the first 20 polarization cycles. We propose these discrepancies reflect that the influence of covalently tethered ligands is more nuanced than simple electronic effects and that their impact may vary depending on the nature of the catalyst's surface chemistry and the density of grafted aryl groups. For example, the documented active site for $\mathrm{MoS}_{2}$ is the edge atoms of the 2D sheet while the basal plane surface is relatively inert for HER. Thus, it is possible that functional groups that bond at the basal plane surface do not have added steric effects because of spatial separation from the catalytically active site. As we show below, that is not the case in $\mathrm{Ni}_{2} \mathrm{P}$ nanocrystals, for which the aryl functional groups bond to atoms directly adjacent to active sites. This could allow for the same functional group to result in different effects based on the intrinsic reactivity and structure of the underlying material.
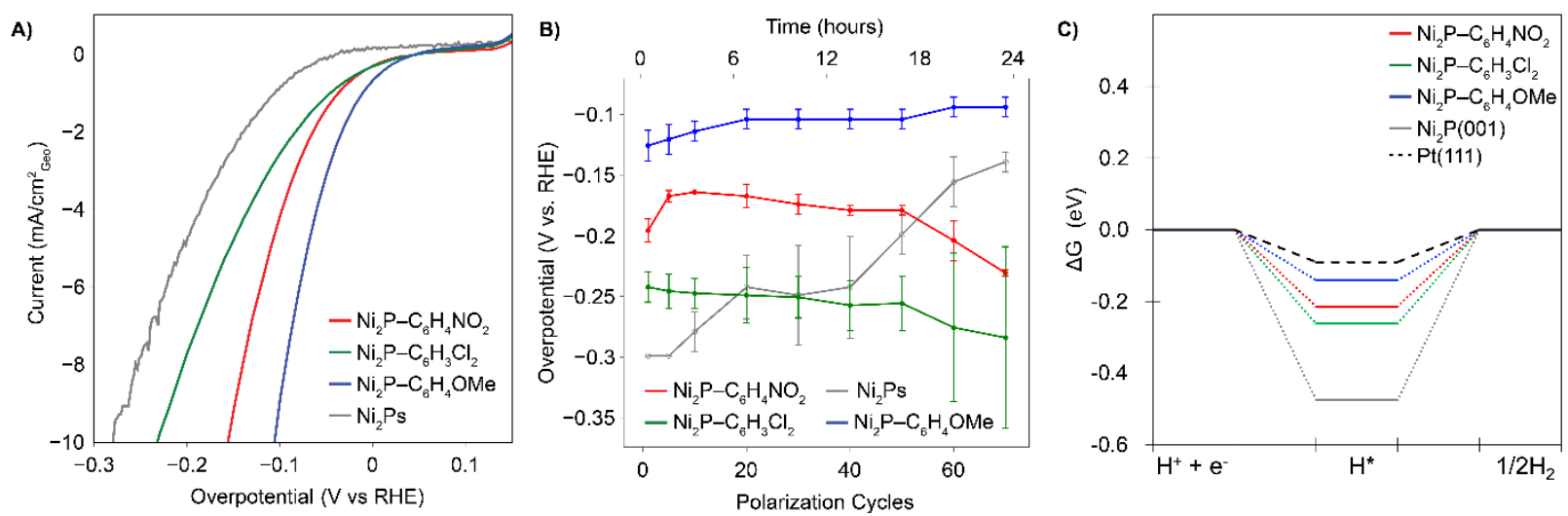

Figure 6. (a) LSV of the $10^{\text {th }}$ polarization cycle for $\mathrm{Ni}_{2} \mathrm{P}$ and Hammett series electrodes collected at $5 \mathrm{mVs}^{-1}$. (b) Overpotential at $10 \mathrm{~mA} / \mathrm{cm}^{2}$ averaged across three separate electrodes for each sample over the course of 24 hours and 75 polarization cycles. (c) Calculated free energy profile at $0.00 \mathrm{~V}, \mathrm{pH}=0$ and $300 \mathrm{~K}$ for the adsorption of $\mathrm{H}$ onto the $\mathrm{Ni}_{2} \mathrm{P}(0001) \mathrm{Ni}_{3}$ hollow site, with and without surface functional groups.

For efficient hydrogen evolution, the hydrogen adsorption free energy should be slightly negative, so that there is a driving force for hydrogen to adsorb onto the catalyst surface, but the adsorption energy is low enough to allow for the subsequent proton coupling steps. As can be seen from Fig. 6C, DFT calculations predict that the free energy for hydrogen adsorption at the $\mathrm{Ni}_{2} \mathrm{P}(0001)$ Ni 3-fold hollow site (possible surface binding sites are shown in Fig. S21) can be modified substantially with surface functionalization of diazonium salts. The trend follows: $\mathrm{Ni}_{2} \mathrm{P}(-$ $0.47 \mathrm{eV})>\mathrm{Ni}_{2} \mathrm{P}_{-} \mathrm{C}_{6} \mathrm{H}_{3} \mathrm{Cl}_{2}(-0.26 \mathrm{eV})>\mathrm{Ni}_{2} \mathrm{P}-\mathrm{C}_{6} \mathrm{H}_{4} \mathrm{NO}_{2}(-0.22 \mathrm{eV})>\mathrm{Ni}_{2} \mathrm{P}-\mathrm{C}_{6} \mathrm{H}_{4} \mathrm{OMe}(-0.14 \mathrm{eV})$, which is in agreement with our experimental findings. In turn, this suggests that the adsorption energies of $\mathrm{H}$ on the nanocrystal surface do not directly follow the electrostatic trend. 
A)
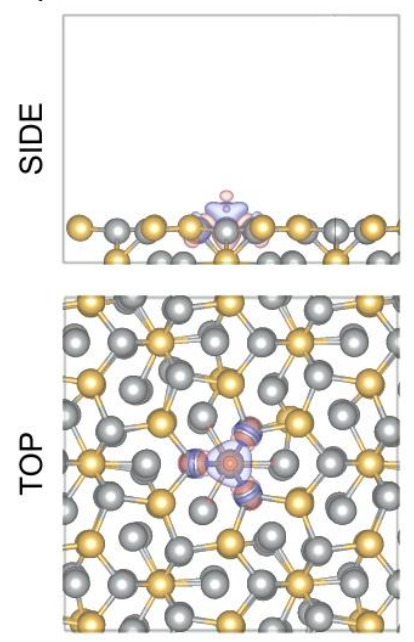

B)
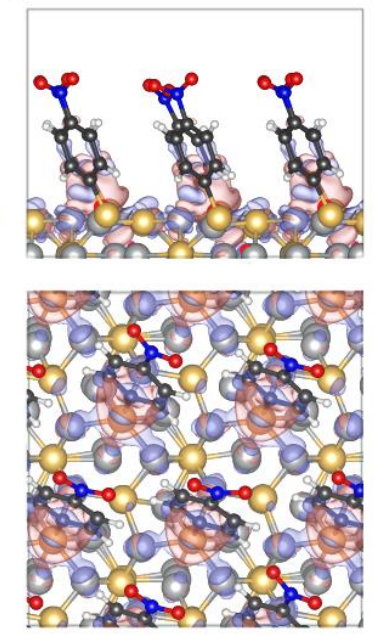

C)
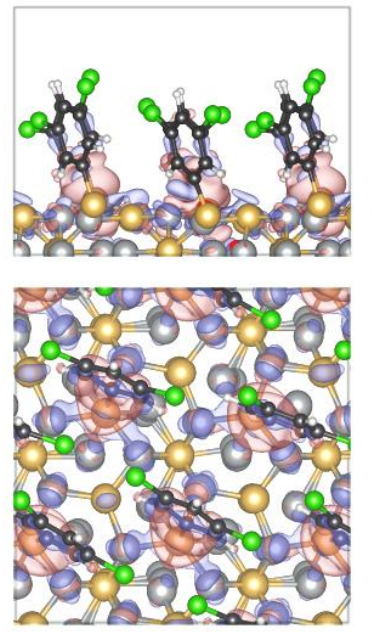

D)
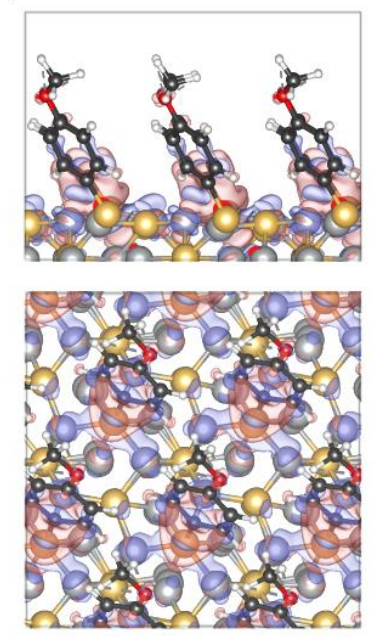

Figure 7. Charge density difference plots highlighting the interactions of key species at the $\mathrm{Ni}_{2} \mathrm{P}(0001)$ interface. (a) $\mathrm{H}$ adsorbed at the $\mathrm{Ni}_{3}$ hollow site. (b) $\mathrm{C}_{6} \mathrm{H}_{4}-\mathrm{NO}_{2}$, (c) $\mathrm{C}_{6} \mathrm{H}_{3}-\mathrm{Cl}_{2}$ and (d) $\mathrm{C}_{6} \mathrm{H}_{4}-\mathrm{OMe}$ adsorbed on the $\mathrm{P}$ top sites. Red and blue shading represent electron depletion and electron accumulation respectively. The iso-surface level is set to $0.013 \mathrm{e} / \AA^{3}$.

To further probe the origins of the observed trend, we examined the effects of electron transfer between the nanocrystal atoms and surface adsorbates by calculating their charge density difference (CDD) (Fig. 7). Fig. 7A shows, that once adsorbed on the surface, hydrogen accumulates electron density from nearby $\mathrm{Ni}$ atoms, forming a surface hydride. Interestingly, from Figs. 7B to 7D, adsorption of diazonium salts on $\mathrm{P}$ top sites exhibit non-local charge redistribution at the Niz hollow site. The net effect of functionalization is the tuning of the hydrogen adsorption free energy at the $\mathrm{Ni}_{3}$ hollow site via electron redistribution resulting in a weaker adsorption energy for $\mathrm{H}$ atoms, thus making the catalytic landscape more thermoneutral relative to the bare surface. These local fluctuations in $\mathrm{Ni}$ charge density can facilitate $\mathrm{H}$ atom diffusion onto the $\mathrm{Ni}$-Ni bridge site (Figs. S27 to S28). The Ni-Ni bridge site is expected to be a catalytically active site in computational studies but is generally only accessed at higher $\mathrm{H}$ coverages. ${ }^{40,117} \mathrm{We}$ further note that the transition from a surface hollow site to lower coordination sites would be hindered by the Pauli repulsion exhibited by the $\mathrm{Cl}$ atoms of the $\mathrm{C}_{6} \mathrm{H}_{3}-\mathrm{Cl}_{2}$ functional groups and the hydride nature of adsorbed $\mathrm{H}\left(\mathrm{H}^{\delta_{-}-} \mathrm{Cl}^{\delta^{-}}\right)$. Additionally, this charge redistribution depends strongly on the alignment of diazonium species above the $\mathrm{P}$ top site. Most noticeable is the repulsive interactions exhibited by $\mathrm{C}_{6} \mathrm{H}_{3} \mathrm{Cl}_{2}$ (Fig. 7C), which is reflected in the binding energy discussed previously. The $\mathrm{Cl}^{\delta_{-}-}-\mathrm{Cl}^{\delta^{-}}$repulsion results in a staggered array of $\mathrm{C}_{6} \mathrm{H}_{3} \mathrm{Cl}_{2}$ species and a disfavored alignment of the $\mathrm{C}_{z}$-orbitals with $\mathrm{P} 3 p$-states. To compensate for this, some $\mathrm{P}$ atoms are pulled away from the surface plane, resulting in significant distortion and a weaker interaction with the surface atoms. The trend in P-atom distortions $\mathrm{C}_{6} \mathrm{H}_{3}-\mathrm{Cl}_{2}(0.57 \AA)>\mathrm{C}_{6} \mathrm{H}_{4}-\mathrm{NO}_{2}(0.31 \AA)>\mathrm{C}_{6} \mathrm{H}_{4}-\mathrm{OMe}(0.29 \AA)$ suggests that para substitution minimizes adsorbate-adsorbate interactions leading to an optimal surface packing of diazonium salts relative to the meta substituted functional groups. Overall, we find that the local electronic structure and bonding interactions of the diazonium salts influence the charge density across the surface, which we hypothesize being the cause of our observed trend. 


\section{Electrocatalytic Stability.}

The next key finding is the variation of activity over multiple polarization cycles. The first and most important point is that the trend in activity induced by the functional groups is held over 24 hours of exposure to catalytic conditions. We propose this is strong evidence in favor of covalent functional groups being a viable pathway to stable hybrid nanomaterials. Another interesting point is the variability, or lack thereof, in activity over time. We observed a gradual increase in activity after successive polarizations for the ligand stripped $\mathrm{Ni}_{2} \mathrm{P}$ sample, as shown in Fig. 6B. This is presumably due to the removal of remnant oleylamine from the surface, allowing for a better wetting of the electrolyte as previously demonstrated in our lab. ${ }^{56}$ However, we cannot count out the possibility of surface reconstruction, which has been theoretically predicted and experimentally measured to take place during catalysis. ${ }^{40,42,118-120}$ If, after prolonged cycling, the dominant surface facet is no longer the $\mathrm{Ni}_{3} \mathrm{P}_{2}$ (0001) termination it could account for the gradual disagreement with the predicted thermodynamic landscape that we observe in earlier cycles (Fig. 6C). Further, this swing in activity is quite large, showing an improvement of $150 \mathrm{mV}$ over the course of our measurements. This contrasts with the functionalized samples which show very little variability under the same conditions, reflecting a maximum average deviation of only $25 \mathrm{mV}$. We note that although the activity of the $\mathrm{Ni}_{2} \mathrm{P}$ electrodes did improve over time, they never achieved higher current densities or lower overpotential than the $\mathrm{Ni}_{2} \mathrm{P}-\mathrm{C}_{6} \mathrm{H}_{4} \mathrm{OMe}$ sample, even in the few samples we measured out to 100 polarization cycles (Fig. S19, S20). Another practically relevant observation during our analysis was the I-V response during cycling. Consistently, we observed wild variations in the current response from $\mathrm{Ni}_{2} \mathrm{P}$ electrodes, presumably due to the formation of hydrogen bubbles. This is reflected in the noisy LSV reported in Fig. 6A but becomes even more apparent at high current densities (Fig. S20). Finally, we were able to observe the retention of key atomic handles by SEM-EDS after repeated cycling, presented in Fig. 8. Critically, this illustrates that the chlorine atoms from the 3,5-dichlorobenzene functional group remain observable and are specifically observed in regions of the image where $\mathrm{Ni}$ and $\mathrm{P}$ are also detected, rather than randomly scattered across the sample.

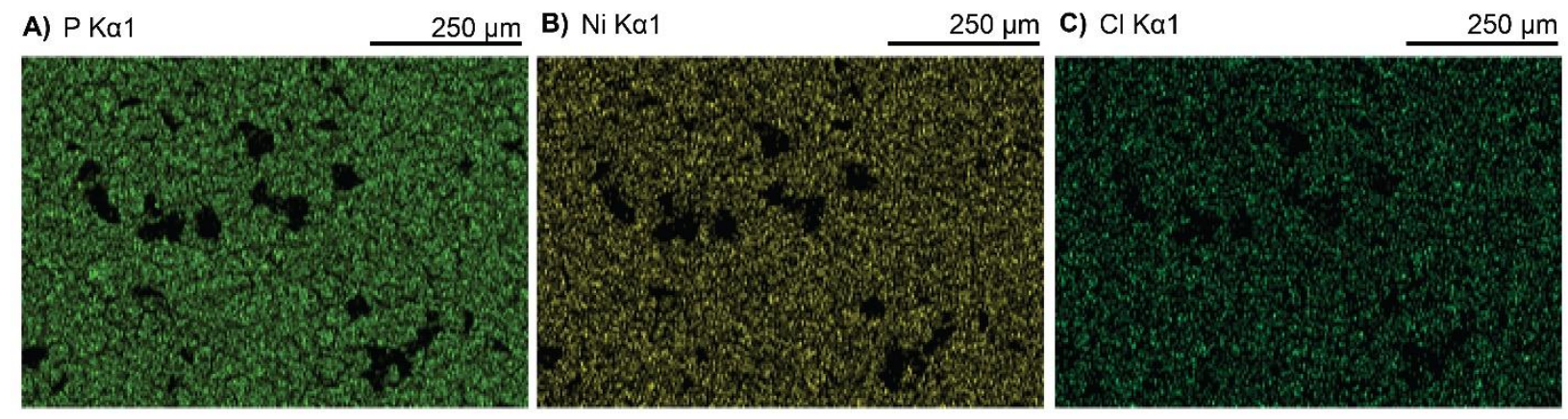

Figure 8. SEM-EDS map of $\mathrm{Ni}_{2} \mathrm{P}-\mathrm{C}_{6} \mathrm{H}_{3} \mathrm{Cl}_{2}$ electrode after 100 polarization cycles showing the presence and spatial overlap of $\mathrm{P}(\mathrm{a}), \mathrm{Ni}(\mathrm{b})$, and $\mathrm{Cl}$ (c) on the electrode after cycling. 


\section{Conclusions and Outlook}

In conclusion, we have formulated synthetic conditions for generating (sub)monolayer coverages of substituted aryl functional groups covalently bound to the surface of $\mathrm{Ni}_{2} \mathrm{P}$ nanocrystals and measured the resultant effects on the nanomaterial's electronic and electrocatalytic properties.

Through our study we have highlighted the need for further investigation into soft ligand removal strategies for colloidal metal phosphide nanocrystals. We have shown that native ligands can participate in side reactions with functionalization precursors and convolute characterization. Still, we show that $\mathrm{Ni}_{2} \mathrm{P}$ nanocrystals can be spontaneously functionalized by substituted aryldiazonium salts. Though we did not observe explicit experimental evidence, computation modeling suggests this functionalization occurs via formation of covalent $\mathrm{P}-\mathrm{C}$ bonds. The degree of functionalization is dictated by the electron density in the aryl ring, where substituents that withdraw more electron density result in higher functionalization densities. We find that the electron donating/withdrawing nature of the surface functional group systematically alters corelevel electron density and the bulk Fermi-level of the materials. Interestingly, we find that the influence on electrocatalytic activity is improved by the addition of substituted aryl groups, but the trend does not clearly track the electrostatic influence of the substituent functional groups. Though unexpected, these findings are validated by DFT modeling of our system which identifies the same broken trend. Through this model we propose that the functional groups have a nuanced effect on activity, which go beyond simple surface dipole effects, and can alter the binding energetics at active sites through surface deformation and steric repulsion. Further, we find that this trend in altered electrocatalytic activity for the HER is maintained over the course of 24 hours and more than 75 polarization cycles, signaling the promise for covalently bound ligands to be robust to catalytic conditions.

Though sterically rigid aryl functional groups are likely not the key ingredient for making an earth abundant catalyst rival the activity of platinum, the utilization of this well-studied class of surface functionalization agents is a promising starting point. We propose that building models and further refining this relatively simple system will lay the groundwork for the development of synthetic procedures to produce elegant systems of covalently tethered proton relay ligands that fully utilize secondary and outer coordination sphere effects. However, there is still much work to be done as a community before such a system can be conceived. For example, exploring the range of electro/nucleophilicities required to form bonds with surface atoms so we can move away from harsh and difficult-to-control radical chemistry. These ideas lead to a further question: would we install an already synthesized complex ligand on the surface, or build it from the bottom-up using post-synthetic transformations once a suitable reactive fragment is bound to the particle surface? Surendranath and co-workers have covalently tethered organometallic catalysts to the edges and defect sites of carbon materials. ${ }^{121,122}$ In those systems, the authors were able to leverage native $o$ quinone moieties that irreversibly condense with diamines to form their covalent linkages. Though such native defects are not as well documented in nanocrystalline phosphide or other more complex materials, a better understanding of the chemical nature of these materials' surface atoms should allow for the development of more elegant functionalization strategies. In fact, collaborative studies between our group and the Mayer group have taken aim at building a more molecular understanding of colloidal metal phosphide nanocrystals. ${ }^{123}$ This concept and line of study will directly bolster our on-going efforts to refine surface functionalization procedures and create better defined hybrid nanomaterials. 


\section{Acknowledgements}

This work was supported as part of the Center for Molecular Electrocatalysis, an Energy Frontier Research Center funded by the U.S. Department of Energy, Office of Science, Office of Basic Energy Sciences. Part of this work was conducted at the Molecular Analysis Facility, a National Nanotechnology Coordinated Infrastructure (NNCI) site at the University of Washington, which is supported in part by funds from the National Science Foundation (awards NNCI-2025489, NNCI-1542101), the Molecular Engineering \& Sciences Institute, and the Clean Energy Institute. Computational resources were provided by the National Energy Research Computing Center (NERSC) at the Lawrence Berkeley National Laboratory and W. R. Wiley Environmental Molecular Sciences Laboratory (EMSL), a national scientific user facility sponsored by the Department of Energy's Office of Biological and Environmental Research located at Pacific Northwest National Laboratory.

\section{References}

(1) Roser, M. Why Did Renewables Become so Cheap so Fast? And What Can We Do to Use This Global Opportunity for Green Growth? Our World in Data. 2020. https://ourworldindata.org/cheap-renewables-growth

(2) Haldorsen, H. H.; Leach, P. Energy 360: Invited Perspective: The Outlook for Energy: A View to 2040. J. Pet. Technol. 2015, 67, 14-19. https://doi.org/10.2118/0415-0014-jpt.

(3) Geels, F. W.; Sovacool, B.; Schwanen, T.; Sorrell, S. Accelerating Innovation Is as Important as Climate Policy. Policy Forum 2017, 357, 1242-1244.

(4) De Luna, P.; Hahn, C.; Higgins, D.; Jaffer, S. A.; Jaramillo, T. F.; Sargent, E. H. What Would It Take for Renewably Powered Electrosynthesis to Displace Petrochemical Processes? Science 2019, 364, eeav3506. https://doi.org/10.1126/science.aav3506.

(5) Seh, Z. W.; Kibsgaard, J.; Dickens, C. F.; Chorkendorff, I.; Nørskov, J. K.; Jaramillo, T. F. Combining Theory and Experiment in Electrocatalysis: Insights into Materials Design. Science 2017, 355, eaad4998. https://doi.org/10.1126/science.aad4998.

(6) Hammer, B.; Norskov, J. K. Why Gold Is the Noblest of All the Metals. Nature 1995, 376, 238-240. https://doi.org/10.1038/376238a0.

(7) Trasatti, S. Work Function, Electronegativity, and Electrochemical Behaviour of Metals. III. Electrolytic Hydrogen Evolution in Acid Solutions. J. Electroanal. Chem. 1972, 39, 163-184. https://doi.org/10.1016/S0022-0728(72)80485-6.

(8) Hammer, B.; Nørskov, J. K. Electronic Factors Determining the Reactivity of Metal Surfaces. Surf. Sci. 1995, 343, 211-220. https://doi.org/10.1016/0039-6028(96)80007-0.

(9) Raugei, S.; Dubois, D. L.; Rousseau, R.; Chen, S.; Ho, M. H.; Bullock, R. M.; Dupuis, M. Toward Molecular Catalysts by Computer. Acc. Chem. Res. 2015, 48, 248-255. https://doi.org/10.1021/ar500342g.

(10) Hammes-Schiffer, S. Controlling Electrons and Protons through Theory: Molecular Electrocatalysts to Nanoparticles. Acc. Chem. Res. 2018, 51, 1975-1983. https://doi.org/10.1021/acs.accounts.8b00240.

(11) and, Z.-P. L.; Hu*, P. General Rules for Predicting Where a Catalytic Reaction Should Occur on Metal Surfaces: A Density Functional Theory Study of $\mathrm{C}-\mathrm{H}$ and $\mathrm{C}-\mathrm{O}$ Bond Breaking/Making on Flat, Stepped, and Kinked Metal Surfaces. J. Am. Chem. Soc. 2003, 125, 1958-1967. https://doi.org/10.1021/JA0207551.

(12) Trasatti, S. Work Function, Electronegativity, and Electrochemical Behaviour of Metals. 
II. Potentials of Zero Charge and "Electrochemical" Work Functions. J. Electroanal. Chem. 1971, 33, 351-378. https://doi.org/10.1016/S0022-0728(71)80123-7.

(13) Quaino, P.; Juarez, F.; Santos, E.; Schmickler, W. Volcano Plots in Hydrogen Electrocatalysis-Uses and Abuses. Beilstein J. Nanotechnol. 2014, 5, 846-854. https://doi.org/10.3762/bjnano.5.96.

(14) Hansen, J. N.; Prats, H.; Toudahl, K. K.; Mørch Secher, N.; Chan, K.; Kibsgaard, J.; Chorkendorff, I. Is There Anything Better than Pt for HER? ACS Energy Lett. 2021, 6 , 1175-1180. https://doi.org/10.1021/acsenergylett.1c00246.

(15) Solis, B. H.; Hammes-Schiffer, S. Proton-Coupled Electron Transfer in Molecular Electrocatalysis: Theoretical Methods and Design Principles. Inorg. Chem. 2014, 53, 6427-6443. https://doi.org/10.1021/ic5002896.

(16) Tributsch, H.; Bennett, J. C. Electrochemistry and Photochemistry of MoS2 Layer Crystals. I. J. Electroanal. Chem. 1977, 81, 97-111. https://doi.org/10.1016/S00220728(77)80363-X.

(17) Benck, J. D.; Hellstern, T. R.; Kibsgaard, J.; Chakthranont, P.; Jaramillo, T. F. Catalyzing the Hydrogen Evolution Reaction (HER) with Molybdenum Sulfide Nanomaterials. ACS Catal. 2014, 4, 3957-3971. https://doi.org/10.1021/cs500923c.

(18) Lukowski, M. A.; Daniel, A. S.; Meng, F.; Forticaux, A.; Li, L.; Jin, S. Enhanced Hydrogen Evolution Catalysis from Chemically Exfoliated Metallic MoS2 Nanosheets. $J$. Am. Chem. Soc. 2013, 135, 10274-10277. https://doi.org/10.1021/ja404523s.

(19) Voiry, D.; Salehi, M.; Silva, R.; Fujita, T.; Chen, M.; Asefa, T.; Shenoy, V. B.; Eda, G.; Chhowalla, M. Conducting MoS2 Nanosheets as Catalysts for Hydrogen Evolution Reaction. Nano Lett. 2013, 13, 6222-6227. https://doi.org/10.1021/nl403661s.

(20) Mundy, M. E.; Ung, D.; Lai, N. L.; Jahrman, E. P.; Seidler, G. T.; Cossairt, B. M. Aminophosphines as Versatile Precursors for the Synthesis of Metal Phosphide Nanocrystals. Chem. Mater. 2018, 30, 5373-5379. https://doi.org/10.1021/acs.chemmater.8b02206.

(21) Layan Savithra, G. H.; Muthuswamy, E.; Bowker, R. H.; Carrillo, B. A.; Bussell, M. E.; Brock, S. L. Rational Design of Nickel Phosphide Hydrodesulfurization Catalysts: Controlling Particle Size and Preventing Sintering. Chem. Mater. 2013, 25, 825-833. https://doi.org/10.1021/cm302680j.

(22) Li, D.; Senevirathne, K.; Aquilina, L.; Brock, S. L. Effect of Synthetic Levers on Nickel Phosphide Nanoparticle Formation: $\mathrm{Ni}_{5} \mathrm{P}_{4}$ and NiP2. Inorg. Chem. 2015, 54, 7968-7975. https://doi.org/10.1021/acs.inorgchem.5b01125.

(23) Muthuswamy, E.; Savithra, G. H. L.; Brock, S. L. Synthetic Levers Enabling Independent Control of Phase, Size, and Morphology in Nickel Phosphide Nanoparticles. ACS Nano 2011, 5, 2402-2411. https://doi.org/10.1021/nn1033357.

(24) Popczun, E. J.; Mckone, J. R.; Read, C. G.; Biacchi, A. J.; Wiltrout, A. M.; Lewis, N. S.; Schaak, R. E. Nanostructured Nickel Phosphide as an Electrocatalyst for the Hydrogen Evolution Reaction. J. Am. Chem. Soc 2013, 135, 9267-9270.

(25) Popczun, E. J.; McKone, J. R.; Read, C. G.; Biacchi, A. J.; Wiltrout, A. M.; Lewis, N. S.; Schaak, R. E. Nanostructured Nickel Phosphide as an Electrocatalyst for the Hydrogen Evolution Reaction. J. Am. Chem. Soc. 2013, 135, 9267-9270. https://doi.org/10.1021/ja403440e.

(26) Callejas, J. F.; Read, C. G.; Roske, C. W.; Lewis, N. S.; Schaak, R. E. Synthesis, Characterization, and Properties of Metal Phosphide Catalysts for the Hydrogen-Evolution 
Reaction. Chem. Mater. 2016, 28, 6017-6044.

https://doi.org/10.1021/acs.chemmater.6b02148.

(27) Popczun, E. J.; Read, C. G.; Roske, C. W.; Lewis, N. S.; Schaak, R. E. Highly Active Electrocatalysis of the Hydrogen Evolution Reaction by Cobalt Phosphide Nanoparticles. Angew. Chem. Int. Ed. 2014, 53, 5427-5430. https://doi.org/10.1002/anie.201402646.

(28) Carenco, S.; Liu, Z.; Salmeron, M. The Birth of Nickel Phosphide Catalysts: Monitoring Phosphorus Insertion into Nickel. ChemCatChem 2017, 9, 2318-2323. https://doi.org/10.1002/cctc.201601526.

(29) Henkes, A. E.; Vasquez, Y.; Schaak, R. E. Converting Metals into Phosphides: A General Strategy for the Synthesis of Metal Phosphide Nanocrystals. J. Am. Chem. Soc. 2007, 129, 1896-1897. https://doi.org/10.1021/ja0685021.

(30) Eagle, F. W.; Rivera-Maldonado, R. A.; Cossairt, B. M. Surface Chemistry of Metal Phosphide Nanocrystals. Annu. Rev. Mater. Res. 2021, 51, 1-24.

https://doi.org/10.1146/annurev-matsci-080819-011036.

(31) Kibsgaard, J.; Tsai, C.; Chan, K.; Benck, J. D.; Nørskov, J. K.; Jaramillo, T. F.; AbildPedersen, F.; Jaramillo, T. F. Designing an Improved Transition Metal Phosphide Catalyst for Hydrogen Evolution Using Experimental and Theoretical Trends. Energy Environ. Sci. 2015, 8, 3022-3029. https://doi.org/10.1039/c5ee02179k.

(32) Man, H. W.; Tsang, C. S.; Li, M. M. J.; Mo, J.; Huang, B.; Lee, L. Y. S.; Leung, Y. chung; Wong, K. Y.; Tsang, S. C. E. Tailored Transition Metal-Doped Nickel Phosphide Nanoparticles for the Electrochemical Oxygen Evolution Reaction (OER). Chem. Commun. 2018, 54, 8630-8633. https://doi.org/10.1039/c8cc03870h.

(33) Liu, J.; Wang, Z.; David, J.; Llorca, J.; Li, J.; Yu, X.; Shavel, A.; Arbiol, J.; Meyns, M.; Cabot, A. Colloidal Ni2-xCoxP Nanocrystals for the Hydrogen Evolution Reaction. $J$. Mater. Chem. A 2018, 6, 11453-11462. https://doi.org/10.1039/c8ta03485k.

(34) El-Refaei, S. M.; Russo, P. A.; Pinna, N. Recent Advances in Multimetal and Doped Transition-Metal Phosphides for the Hydrogen Evolution Reaction at Different PH Values. ACS Appl. Mater. Interf. 2021, 13, 22077-22097. https://doi.org/10.1021/acsami.1c02129.

(35) Hitihami-Mudiyanselage, A.; Senevirathne, K.; Brock, S. L. Bottom-Up Assembly of Ni 2 P Nanoparticles into Three-Dimensional Architectures: An Alternative Mechanism for Phosphide Gelation. Chem. Mater. 2014, 26, 6251-6256. https://doi.org/10.1021/cm5030958.

(36) Yu, X.; Yu, Z. Y.; Zhang, X. L.; Zheng, Y. R.; Duan, Y.; Gao, Q.; Wu, R.; Sun, B.; Gao, M. R.; Wang, G.; Yu, S. H. "Superaerophobic" Nickel Phosphide Nanoarray Catalyst for Efficient Hydrogen Evolution at Ultrahigh Current Densities. J. Am. Chem. Soc. 2019, 141, 7537-7543. https://doi.org/10.1021/jacs.9b02527.

(37) Li, S.-H.; Qi, M.-Y.; Tang, Z.-R.; Xu, Y.-J. Nanostructured Metal Phosphides: From Controllable Synthesis to Sustainable Catalysis. Chem. Soc. Rev. 2021, 50, 7539-7586. https://doi.org/10.1039/d1cs00323b.

(38) Liu, P.; Rodriguez, J. A. Catalysts for Hydrogen Evolution from the [NiFe] Hydrogenase to the Ni2P(001) Surface: The Importance of Ensemble Effect. J. Am. Chem. Soc. 2005, 127, 14871-14878. https://doi.org/10.1021/ja0540019.

(39) Hansen, M. H.; Stern, L. A.; Feng, L.; Rossmeisl, J.; Hu, X. Widely Available Active Sites on Ni2P for Electrochemical Hydrogen Evolution - Insights from First Principles Calculations. Phys. Chem. Chem. Phys. 2015, 17, 10823-10829. 
https://doi.org/10.1039/c5cp01065a.

(40) Wexler, R. B.; Martirez, J. M. P.; Rappe, A. M. Active Role of Phosphorus in the Hydrogen Evolving Activity of Nickel Phosphide (0001) Surfaces. ACS Catal. 2017, 7, 7718-7725. https://doi.org/10.1021/acscatal.7b02761.

(41) Wexler, R. B.; Martirez, J. M. P.; Rappe, A. M. Stable Phosphorus-Enriched (0001) Surfaces of Nickel Phosphides. Chem. Mater. 2016, 28, 5365-5372.

https://doi.org/10.1021/acs.chemmater.6b01437.

(42) Wexler, R. B.; Martirez, J. M. P.; Rappe, A. M. Chemical Pressure-Driven Enhancement of the Hydrogen Evolving Activity of Ni2P from Nonmetal Surface Doping Interpreted via Machine Learning. J. Am. Chem. Soc. 2018, 140, 4678-4683. https://doi.org/10.1021/jacs.8b00947.

(43) Rice, P. S.; Hu, P. Understanding Supported Noble Metal Catalysts Using First-Principles Calculations. J. Chem. Phys. 2019, 180902. https://doi.org/10.1063/1.5126090.

(44) Guharoy, U.; Ramirez Reina, T.; Olsson, E.; Gu, S.; Cai, Q. Theoretical Insights of Ni2P (0001) Surface toward Its Potential Applicability in CO2 Conversion via Dry Reforming of Methane. ACS Catal. 2019, 9, 3487-3497. https://doi.org/10.1021/acscatal.8b04423.

(45) Chen, S.; Rousseau, R.; Raugei, S.; Dupuis, M.; DuBois, D. L.; Bullock, R. M. Comprehensive Thermodynamics of Nickel Hydride Bis(Diphosphine) Complexes: A Predictive Model through Computations. Organometallics 2011, 30, 6108-6118. https://doi.org/10.1021/om200645x.

(46) Wiedner, E. S. Thermodynamic Hydricity of [FeFe]-Hydrogenases. J. Am. Chem. Soc. 2019, 141, 7212-7222. https://doi.org/10.1021/jacs.8b13084.

(47) Wiedner, E. S.; Chambers, M. B.; Pitman, C. L.; Bullock, R. M.; Miller, A. J. M.; Appel, A. M. Thermodynamic Hydricity of Transition Metal Hydrides. Chem. Rev. 2016, 116, 8655-8692. https://doi.org/10.1021/acs.chemrev.6b00168.

(48) McCarthy, B. D.; Dempsey, J. L. Decoding Proton-Coupled Electron Transfer with Potential-PKa Diagrams. Inorg. Chem. 2017, 56, 1225-1231. https://doi.org/10.1021/acs.inorgchem.6b02325.

(49) Helm, M. L.; Stewart, M. P.; Bullock, R. M.; DuBois, M. R.; Dubois, D. L. A Synthetic Nickel Electrocatalyst with a Turnover Frequency Above 100,000 S-1 for H2 Production. Science 2011, 333, 863-866. https://doi.org/10.1126/science.1205864.

(50) O’Hagan, M.; Shaw, W. J.; Raugei, S.; Chen, S.; Yang, J. Y.; Kilgore, U. J.; Dubois, D. L.; Bullock, R. M. Moving Protons with Pendant Amines: Proton Mobility in a Nickel Catalyst for Oxidation of Hydrogen. J. Am. Chem. Soc. 2011, 133, 14301-14312. https://doi.org/10.1021/ja201838x.

(51) Liao, Q.; Liu, T.; Johnson, S. I.; Klug, C. M.; Wiedner, E. S.; Morris Bullock, R.; Dubois, D. L. Evaluation of Attractive Interactions in the Second Coordination Sphere of Iron Complexes Containing Pendant Amines. Dalt. Trans. 2019, 48, 4867-4878. https://doi.org/10.1039/c9dt00708c.

(52) Wang, Y. H.; Schneider, P. E.; Goldsmith, Z. K.; Mondal, B.; Hammes-Schiffer, S.; Stahl, S. S. Brønsted Acid Scaling Relationships Enable Control over Product Selectivity from O2 Reduction with a Mononuclear Cobalt Porphyrin Catalyst. ACS Cent. Sci. 2019, 5, 1024-1034. https://doi.org/10.1021/acscentsci.9b00194.

(53) Ung, D.; Murphy, I. A.; Cossairt, B. M. Designing Nanoparticle Interfaces for InnerSphere Catalysis. Dalt. Trans. 2020, 49, 4995-5005. https://doi.org/10.1039/d0dt00785d. Rossi, L. M.; Fiorio, J. L.; Garcia, M. A. S.; Ferraz, C. P. The Role and Fate of Capping 
Ligands in Colloidally Prepared Metal Nanoparticle Catalysts. Dalt. Trans. 2018, 47, 5889-5915. https://doi.org/10.1039/c7dt04728b.

(55) Ortunõ, M. A.; López, N. Reaction Mechanisms at the Homogeneous-Heterogeneous Frontier: Insights from First-Principles Studies on Ligand-Decorated Metal Nanoparticles. Catal. Sci. Technol. 2019, 9, 5173-5185. https://doi.org/10.1039/c9cy01351b.

(56) Ung, D.; Cossairt, B. M. Effect of Surface Ligands on CoP for the Hydrogen Evolution Reaction. ACS Appl. Energy Mater. 2019, 2, 1642-1645. https://doi.org/10.1021/acsaem.9b00240.

(57) Henckel, D. A.; Lenz, O.; Cossairt, B. M. Effect of Ligand Coverage on Hydrogen Evolution Catalyzed by Colloidal WSe2. ACS Catal. 2017, 7, 2815-2820. https://doi.org/10.1021/acscatal.7b00074.

(58) Zobel, M.; Neder, R. B.; Kimber, S. A. J. Universal Solvent Restructuring Induced by Colloidal Nanoparticles. Science 2015, 347, 292-294.

https://doi.org/10.1126/science.1261412.

(59) Kroupa, D. M.; Vörös, M.; Brawand, N. P.; McNichols, B. W.; Miller, E. M.; Gu, J.; Nozik, A. J.; Sellinger, A.; Galli, G.; Beard, M. C. Tuning Colloidal Quantum Dot Band Edge Positions through Solution-Phase Surface Chemistry Modification. Nat. Commun. 2017, 8, 15257. https://doi.org/10.1038/ncomms15257.

(60) Brown, P. R.; Kim, D.; Lunt, R. R.; Zhao, N.; Bawendi, M. G.; Grossman, J. C.; Bulović, V. Energy Level Modification in Lead Sulfide Quantum Dot Thin Films through Ligand Exchange. ACS Nano 2014, 8, 5863-5872. https://doi.org/10.1021/nn500897c.

(61) Benson, E. E.; Zhang, H.; Schuman, S. A.; Nanayakkara, S. U.; Bronstein, N. D.; Ferrere, S.; Blackburn, J. L.; Miller, E. M. Balancing the Hydrogen Evolution Reaction, Surface Energetics, and Stability of Metallic MoS2 Nanosheets via Covalent Functionalization. $J$. Am. Chem. Soc. 2018, 140, 441-450. https://doi.org/10.1021/jacs.7b11242.

(62) Anderson, N. C.; Hendricks, M. P.; Choi, J. J.; Owen, J. S. Ligand Exchange and the Stoichiometry of Metal Chalcogenide Nanocrystals: Spectroscopic Observation of Facile Metal-Carboxylate Displacement and Binding. J. Am. Chem. Soc. 2013, 135, 1853618548. https://doi.org/10.1021/ja4086758.

(63) Heuer-Jungemann, A.; Feliu, N.; Bakaimi, I.; Hamaly, M.; Alkilany, A.; Chakraborty, I.; Masood, A.; Casula, M. F.; Kostopoulou, A.; Oh, E.; Susumu, K.; Stewart, M. H.; Medintz, I. L.; Stratakis, E.; Parak, W. J.; Kanaras, A. G. The Role of Ligands in the Chemical Synthesis and Applications of Inorganic Nanoparticles. Chem. Rev. 2019, 119, 4819-4880. https://doi.org/10.1021/acs.chemrev.8b00733.

(64) Hassinen, A.; Moreels, I.; De Nolf, K.; Smet, P. F.; Martins, J. C.; Hens, Z. Short-Chain Alcohols Strip X-Type Ligands and Quench the Luminescence of PbSe and CdSe Quantum Dots, Acetonitrile Does Not. J. Am. Chem. Soc. 2012, 134, 20705-20712. https://doi.org/10.1021/ja308861d.

(65) Ritchhart, A.; Cossairt, B. M. Quantifying Ligand Exchange on InP Using an Atomically Precise Cluster Platform. Inorg. Chem. 2019, 58, 2840-2847. https://doi.org/10.1021/acs.inorgchem.8b03524.

(66) Knauf, R. R.; Lennox, J. C.; Dempsey, J. L. Quantifying Ligand Exchange Reactions at CdSe Nanocrystal Surfaces. Chem. Mater. 2016, 28, 4762-4770. https://doi.org/10.1021/acs.chemmater.6b01827.

(67) Sluydts, M.; De Nolf, K.; Van Speybroeck, V.; Cottenier, S.; Hens, Z. Ligand Addition Energies and the Stoichiometry of Colloidal Nanocrystals. ACS Nano 2016, 10, 1462- 
1474. https://doi.org/10.1021/acsnano.5b06965.

(68) Nag, A.; Kovalenko, M. V.; Lee, J. S.; Liu, W.; Spokoyny, B.; Talapin, D. V. Metal-Free Inorganic Ligands for Colloidal Nanocrystals: S2-, HS-, Se2-, HSe-, Te2-, HTe -, TeS32-, OH-, and NH 2- as Surface Ligands. J. Am. Chem. Soc. 2011, 133, 10612-10620. https://doi.org/10.1021/ja2029415.

(69) Fan, J. Z.; Andersen, N. T.; Biondi, M.; Todorović, P.; Sun, B.; Ouellette, O.; Abed, J.; Sagar, L. K.; Choi, M. J.; Hoogland, S.; de Arquer, F. P. G.; Sargent, E. H. Mixed Lead Halide Passivation of Quantum Dots. Adv. Mater. 2019, 31, 1904304. https://doi.org/10.1002/adma.201904304.

(70) Hughes, B. K.; Ruddy, D. A.; Blackburn, J. L.; Smith, D. K.; Bergren, M. R.; Nozik, A. J.; Johnson, J. C.; Beard, M. C. Control of PbSe Quantum Dot Surface Chemistry and Photophysics Using an Alkylselenide Ligand. ACS Nano 2012, 6, 5498-5506. https://doi.org/10.1021/nn301405j.

(71) Mohamed, A. A.; Salmi, Z.; Dahoumane, S. A.; Mekki, A.; Carbonnier, B.; Chehimi, M. M. Functionalization of Nanomaterials with Aryldiazonium Salts. Adv. Colloid Interface Sci. 2015, 225, 16-36. https://doi.org/10.1016/j.cis.2015.07.011.

(72) Bouden, S.; Pinson, J.; Vautrin-Ul, C. Electrografting of Diazonium Salts: A Kinetics Study. Electrochem. commun. 2017, 81, 120-123. https://doi.org/10.1016/j.elecom.2017.06.007.

(73) Adenier, A.; Cabet-Deliry, E.; Chaussé, A.; Griveau, S.; Mercier, F.; Pinson, J.; VautrinUl, C. Grafting of Nitrophenyl Groups on Carbon and Metallic Surfaces without Electrochemical Induction. Chem. Mater. 2005, 17, 491-501. https://doi.org/10.1021/cm0490625.

(74) Toupin, M.; Bélanger, D. Spontaneous Functionalization of Carbon Black by Reaction with 4-Nitrophenyldiazonium Cations. Langmuir 2008, 24, 1910-1917. https://doi.org/10.1021/la702556n.

(75) Paulus, G. L. C.; Wang, Q. H.; Strano, M. S. Covalent Electron Transfer Chemistry of Graphene with Diazonium Salts. Acc. Chem. Res. 2013, 46, 160-170. https://doi.org/10.1021/ar300119z.

(76) Strano, M. S.; Dyke, C. A.; Usrey, M. L.; Barone, P. W.; Allen, M. J.; Shan, H.; Kittrell, C.; Hauge, R. H.; Tour, J. M.; Smalley. Electronic Structure Control of Single-Walled Carbon Nanotube Functionalization. Science 2003, 301, 1519-1522. https://doi.org/10.1126/science.1087691.

(77) Allongue, P.; Delamar, M.; Desbat, B.; Fagebaume, O.; Hitmi, R.; Pinson, J.; Savéant, J. M. Covalent Modification of Carbon Surfaces by Aryl Radicals Generated from the Electrochemical Reduction of Diazonium Salts. J. Am. Chem. Soc. 1997, 119, 201-207. https://doi.org/10.1021/ja963354s.

(78) Hirsch, A.; Hauke, F. Post-Graphene 2D Chemistry: The Emerging Field of Molybdenum Disulfide and Black Phosphorus Functionalization. Angew. Chem. Int. Ed. 2018, 57, 4338-4354. https://doi.org/10.1002/anie.201708211.

(79) Knirsch, K. C.; Berner, N. C.; Nerl, H. C.; Cucinotta, C. S.; Gholamvand, Z.; McEvoy, N.; Wang, Z.; Abramovic, I.; Vecera, P.; Halik, M.; Sanvito, S.; Duesberg, G. S.; Nicolosi, V.; Hauke, F.; Hirsch, A.; Coleman, J. N.; Backes, C. Basal-Plane Functionalization of Chemically Exfoliated Molybdenum Disulfide by Diazonium Salts. ACS Nano 2015, 9 , 6018-6030. https://doi.org/10.1021/acsnano.5b00965.

(80) Ryder, C. R.; Wood, J. D.; Wells, S. A.; Yang, Y.; Jariwala, D.; Marks, T. J.; Schatz, G. 
C.; Hersam, M. C. Covalent Functionalization and Passivation of Exfoliated Black Phosphorus via Aryl Diazonium Chemistry. Nat. Chem. 2016, 8, 597-602. https://doi.org/10.1038/nchem.2505.

(81) Giannozzi, P.; Baroni, S.; Bonini, N.; Calandra, M.; Car, R.; Cavazzoni, C.; Ceresoli, D.; Chiarotti, G. L.; Cococcioni, M.; Dabo, I.; Dal Corso, A.; De Gironcoli, S.; Fabris, S.; Fratesi, G.; Gebauer, R.; Gerstmann, U.; Gougoussis, C.; Kokalj, A.; Lazzeri, M.; MartinSamos, L.; Marzari, N.; Mauri, F.; Mazzarello, R.; Paolini, S.; Pasquarello, A.; Paulatto, L.; Sbraccia, C.; Scandolo, S.; Sclauzero, G.; Seitsonen, A. P.; Smogunov, A.; Umari, P.; Wentzcovitch, R. M. QUANTUM ESPRESSO: A Modular and Open-Source Software Project for Quantum Simulations of Materials. J. Phys. Condens. Matter 2009, 21, 395502. https://doi.org/10.1088/0953-8984/21/39/395502.

(82) Perdew, J. P.; Burke, K.; Ernzerhof, M. Generalized Gradient Approximation Made Simple. Phys. Rev. Lett. 1996, 77, 3865-3868. https://doi.org/10.1103/PhysRevLett.77.3865.

(83) Grimme, S. Semiempirical GGA-Type Density Functional Constructed with a LongRange Dispersion Correction. J. Comput. Chem. 2006, 27, 1787-1799. https://doi.org/10.1002/jcc.20495.

(84) Joubert, D. From Ultrasoft Pseudopotentials to the Projector Augmented-Wave Method. Phys. Rev. B - Condens. Matter Mater. Phys. 1999, 59, 1758-1775. https://doi.org/10.1103/PhysRevB.59.1758.

(85) Monkhorst, H. J.; Pack, J. D. Special Points for Brillouin-Zone Integrations. Phys. Rev. B 1976, 13, 5188-5192. https://doi.org/10.1103/PhysRevB.13.5188.

(86) Head, J. D.; Zerner, M. C. A Broyden-Fletcher-Goldfarb-Shanno Optimization Procedure for Molecular Geometries. Chem. Phys. Lett. 1985, 122, 264-270. https://doi.org/10.1016/0009-2614(85)80574-1.

(87) Kawamura, M.; Gohda, Y.; Tsuneyuki, S. Improved Tetrahedron Method for the Brillouin-Zone Integration Applicable to Response Functions. Phys. Rev. B - Condens. Matter Mater. Phys. 2014, 89, 94515. https://doi.org/10.1103/PhysRevB.89.094515.

(88) Buffard, A.; Dreyfuss, S.; Nadal, B.; Heuclin, H.; Xu, X.; Patriarche, G.; Mézailles, N.; Dubertret, B. Mechanistic Insight and Optimization of InP Nanocrystals Synthesized with Aminophosphines. Chem. Mater. 2016, 28, 5925-5934. https://doi.org/10.1021/acs.chemmater.6b02456.

(89) Kim, K.; Yoo, D.; Choi, H.; Tamang, S.; Ko, J. H.; Kim, S.; Kim, Y. H.; Jeong, S. HalideAmine Co-Passivated Indium Phosphide Colloidal Quantum Dots in Tetrahedral Shape. Angew. Chem. Int. Ed. 2016, 55, 3714-3718. https://doi.org/10.1002/anie.201600289.

(90) Lin-Vien, D.; Colthup, N. B.; Fateley, W. G.; Grasselli, J. G. Introduction. In The Handbook of Infrared and Raman Characteristic Frequencies of Organic Molecules; 1991. https://doi.org/10.1016/b978-0-08-057116-4.50007-9.

(91) Mourdikoudis, S.; Liz-Marzán, L. M. Oleylamine in Nanoparticle Synthesis. Chem. Mater. 2013, 25, 1465-1476. https://doi.org/10.1021/cm4000476.

(92) Baranov, D.; Lynch, M. J.; Curtis, A. C.; Carollo, A. R.; Douglass, C. R.; Mateo-Tejada, A. M.; Jonas, D. M. Purification of Oleylamine for Materials Synthesis and Spectroscopic Diagnostics for Trans Isomers. Chem. Mater. 2019, 31, 1223-1230. https://doi.org/10.1021/acs.chemmater.8b04198.

(93) Kessler, M. L.; Starr, H. E.; Knauf, R. R.; Rountree, K. J.; Dempsey, J. L. Exchange Equilibria of Carboxylate-Terminated Ligands at PbS Nanocrystal Surfaces. Phys. Chem. 
Chem. Phys. 2018, 20, 23649-23655. https://doi.org/10.1039/c8cp04275f.

(94) Kuntz, K. L.; Wells, R. A.; Hu, J.; Yang, T.; Dong, B.; Guo, H.; Woomer, A. H.; Druffel, D. L.; Alabanza, A.; Tománek, D.; Warren, S. C. Control of Surface and Edge Oxidation on Phosphorene. ACS Appl. Mater. Interfaces 2017, 9, 9126-9135.

https://doi.org/10.1021/acsami.6b16111.

(95) Briggs, D. X-Ray Photoelectron Spectroscopy (XPS). Handb. Adhes. Second Ed. 2005, 621-622. https://doi.org/10.1002/0470014229.ch22.

(96) Rosen, E. L.; Buonsanti, R.; Llordes, A.; Sawvel, A. M.; Milliron, D. J.; Helms, B. A. Exceptionally Mild Reactive Stripping of Native Ligands from Nanocrystal Surfaces by Using Meerwein's Salt. Angew. Chem. Int. Ed. 2012, 51, 684-689. https://doi.org/10.1002/anie.201105996.

(97) Nelson, A.; Zong, Y.; Fritz, K. E.; Suntivich, J.; Robinson, R. D. Assessment of Soft Ligand Removal Strategies: Alkylation as a Promising Alternative to High-Temperature Treatments for Colloidal Nanoparticle Surfaces. ACS Mater. Lett. 2019, 1, 177-184. https://doi.org/10.1021/acsmaterialslett.9b00089.

(98) Pankhurst, J. R.; Iyengar, P.; Loiudice, A.; Mensi, M.; Buonsanti, R. Metal-Ligand Bond Strength Determines the Fate of Organic Ligands on the Catalyst Surface during the Electrochemical CO2reduction Reaction. Chem. Sci. 2020, 11, 9296-9302. https://doi.org/10.1039/d0sc03061a.

(99) Dong, A.; Ye, X.; Chen, J.; Kang, Y.; Gordon, T.; Kikkawa, J. M.; Murray, C. B. A Generalized Ligand-Exchange Strategy Enabling Sequential Surface Functionalization of Colloidal Nanocrystals. J. Am. Chem. Soc. 2011, 133, 998-1006. https://doi.org/10.1021/ja108948z.

(100) Zollinger, H. Reactivity and Stability of Arenediazonium Ions. Acc. Chem. Res. 1973, 6, 335-341. https://doi.org/10.1021/ar50070a002.

(101) Nandiyanto, A. B. D.; Oktiani, R.; Ragadhita, R. How to Read and Interpret Ftir Spectroscope of Organic Material. Indones. J. Sci. Technol. 2019, 4. https://doi.org/10.17509/ijost.v4i1.15806.

(102) Slovetskii, V. I. IR Spectra of Nitro Compounds. Bull. Acad. Sci. USSR, Div. Chem. Sci. 1971, 19, 2086-2091.

(103) Chen, X.; Denninger, P.; Stimpel-Lindner, T.; Spiecker, E.; Duesberg, G. S.; Backes, C.; Knirsch, K. C.; Hirsch, A. Defect Engineering of Two-Dimensional Molybdenum Disulfide. Chem. Eur. J. 2020, 26, 6535-6544. https://doi.org/10.1002/chem.202000286.

(104) Menanteau, T.; Levillain, E.; Breton, T. Electrografting via Diazonium Chemistry: From Multilayer to Monolayer Using Radical Scavenger. Chem. Mater. 2013, 25, 2905-2909. https://doi.org/10.1021/cm401512c.

(105) Actis, P.; Caulliez, G.; Shul, G.; Opallo, M.; Mermoux, M.; Marcus, B.; Boukherroub, R.; Szunerits, S. Functionalization of Glassy Carbon with Diazonium Salts in Ionic Liquids. Langmuir 2008, 24, 6327-6333. https://doi.org/10.1021/la703714a.

(106) Saby, C.; Ortiz, B.; Champagne, G. Y.; Bélanger, D. Electrochemical Modification of Glassy Carbon Electrode Using Aromatic Diazonium Salts. 1. Blocking Effect of 4Nitrophenyl and 4-Carboxyphenyl Groups. Langmuir 1997, 13, 6805-6813. https://doi.org/10.1021/la961033o.

(107) Zhou, W.; Dong, L.; Tan, L.; Tang, Q. Enhancing the Surface Reactivity of Black Phosphorus on Hydrogen Evolution by Covalent Chemistry. J. Phys. Chem. C 2021, 125, 7581-7589. https://doi.org/10.1021/acs.jpcc.1c01110. 
(108) Chu, X. S.; Yousaf, A.; Li, D. O.; Tang, A. A.; Debnath, A.; Ma, D.; Green, A. A.; Santos, E. J. G.; Wang, Q. H. Direct Covalent Chemical Functionalization of Unmodified TwoDimensional Molybdenum Disulfide. Chem. Mater. 2018, 30, 2112-2128. https://doi.org/10.1021/acs.chemmater.8b00173.

(109) Yan, E. X.; Cabán-Acevedo, M.; Papadantonakis, K. M.; Brunschwig, B. S.; Lewis, N. S. Reductant-Activated, High-Coverage, Covalent Functionalization of $1 \mathrm{~T}^{\prime}$-MoS 2. ACS Mater. Lett. 2020, 2, 133-139. https://doi.org/10.1021/acsmaterialslett.9b00241.

(110) Kariuki, J. K.; McDermott, M. T. Formation of Multilayers on Glassy Carbon Electrodes via the Reduction of Diazonium Salts. Langmuir 2001, 17, 5947-5951. https://doi.org/10.1021/la010415d.

(111) Blanchard, P. E. R.; Grosvenor, A. P.; Cavell, R. G.; Mar, A. Effects of Metal Substitution in Transition-Metal Phosphides (Ni 1-XM'x)2P $\left(\mathrm{M}^{\prime}=\mathrm{Cr}, \mathrm{Fe}, \mathrm{Co}\right)$ Studied by X-Ray Photoelectron and Absorption Spectroscopy. J. Mater. Chem. 2009, 19, 6015-6022. https://doi.org/10.1039/b904250d.

(112) Van Der Heide, P. A. W. Photoelectron Binding Energy Shifts Observed during Oxidation of Group IIA, IIIA and IVA Elemental Surfaces. J. Electron Spectros. Relat. Phenomena 2006, 151, 79-91. https://doi.org/10.1016/j.elspec.2005.11.001.

(113) Voiry, D.; Chhowalla, M.; Gogotsi, Y.; Kotov, N. A.; Li, Y.; Penner, R. M.; Schaak, R. E.; Weiss, P. S. Best Practices for Reporting Electrocatalytic Performance of Nanomaterials. ACS Nano 2018, 12, 9635-9638. https://doi.org/10.1021/acsnano.8b07700.

(114) Aziz, S. T.; Kumar, S.; Riyajuddin, S.; Ghosh, K.; Nessim, G. D.; Dubal, D. P. Bimetallic Phosphides for Hybrid Supercapacitors. J. Phys. Chem. Lett. 2021, 12, 5138-5149. https://doi.org/10.1021/acs.jpclett.1c00562.

(115) Li, L.; Mai, W.; Lu, J.; Zhou, W.; Zhou, K.; Tang, Z.; Yang, L.; Cheng, S.; Chen, S. Ultrahigh-Performance Pseudocapacitor Electrodes Based on Transition Metal Phosphide Nanosheets Array via Phosphorization: A General and Effective Approach. Adv. Funct. Mater. 2015, 25, 7530-7538. https://doi.org/10.1002/adfm.201503662.

(116) Trasatti, S.; Petrii, O. A. Real Surface Area Measurements in Electrochemistry. $J$. Electroanal. Chem. 1992, 327, 353-376. https://doi.org/10.1016/0926-860x(96)80148-7.

(117) Wang, G.; Shi, Y.; Mei, J.; Xiao, C.; Hu, D.; Chi, K.; Gao, S.; Duan, A.; Zheng, P. DFT Insights into Hydrogen Activation on the Doping Ni2P Surfaces under the Hydrodesulfurization Condition. Appl. Surf. Sci. 2021, 538, 148160. https://doi.org/10.1016/j.apsusc.2020.148160.

(118) Laursen, A. B.; Wexler, R. B.; Whitaker, M. J.; Izett, E. J.; Calvinho, K. U. D.; Hwang, S.; Rucker, R.; Wang, H.; Li, J.; Garfunkel, E.; Greenblatt, M.; Rappe, A. M.; Dismukes, G. C. Climbing the Volcano of Electrocatalytic Activity While Avoiding Catalyst Corrosion: Ni3P, a Hydrogen Evolution Electrocatalyst Stable in Both Acid and Alkali. ACS Catal. 2018, 8, 4408-4419. https://doi.org/10.1021/acscatal.7b04466.

(119) Saadi, F. H.; Carim, A. I.; Verlage, E.; Hemminger, J. C.; Lewis, N. S.; Soriaga, M. P. $\mathrm{CoP}$ as an Acid-Stable Active Electrocatalyst for the Hydrogen-Evolution Reaction: Electrochemical Synthesis, Interfacial Characterization and Performance Evaluation. $J$. Phys. Chem. C 2014, 118, 29294-29300. https://doi.org/10.1021/jp5054452.

(120) Saadi, F. H.; Carim, A. I.; Drisdell, W. S.; Gul, S.; Baricuatro, J. H.; Yano, J.; Soriaga, M. P.; Lewis, N. S. Operando Spectroscopic Analysis of CoP Films Electrocatalyzing the Hydrogen-Evolution Reaction. J. Am. Chem. Soc. 2017, 139, 12927-12930. 
https://doi.org/10.1021/jacs.7b07606.

(121) Jackson, M. N.; Oh, S.; Kaminsky, C. J.; Chu, S. B.; Zhang, G.; Miller, J. T.;

Surendranath, Y. Strong Electronic Coupling of Molecular Sites to Graphitic Electrodes via Pyrazine Conjugation. J. Am. Chem. Soc. 2018, 140, 1004-1010. https://doi.org/10.1021/jacs.7b10723.

(122) Oh, S.; Gallagher, J. R.; Miller, J. T.; Surendranath, Y. Graphite-Conjugated Rhenium Catalysts for Carbon Dioxide Reduction. J. Am. Chem. Soc. 2016, 138, 1820-1823. https://doi.org/10.1021/jacs.5b13080.

(123) Delley, M. F.; Wu, Z.; Mundy, M. E.; Ung, D.; Cossairt, B. M.; Wang, H.; Mayer, J. M. Hydrogen on Cobalt Phosphide. J. Am. Chem. Soc. 2019, 141, 15390-15402. https://doi.org/10.1021/jacs.9b07986. 